\title{
Top-down effects on early visual processing in humans: a predictive coding framework
}

Karsten Rauss $^{1,2,3^{*}}$, Sophie Schwartz ${ }^{1,2,4}$ \& Gilles Pourtois ${ }^{5}$

${ }^{1}$ Department of Neuroscience, University of Geneva, Switzerland

${ }^{2}$ Geneva Neuroscience Center, University of Geneva, Switzerland

${ }^{3}$ Institute of Medical Psychology and Behavioral Neurobiology, University of Tübingen, Germany

${ }^{4}$ Swiss Center for Affective Sciences, University of Geneva, Switzerland

${ }^{5}$ Department of Experimental Clinical and Health Psychology, Ghent University, Belgium

*corresponding author, present address: Institute of Medical Psychology and Behavioral

Neurobiology, University of Tübingen, Germany; karsten.rauss@ uni-tuebingen.de

Running head:

Top-down effects and $\mathrm{C} 1$ 


\begin{abstract}
An increasing number of human electroencephalography (EEG) studies examining the earliest component of the visual evoked potential, the so-called $\mathrm{C} 1$, have cast doubts on the previously prevalent notion that this component is impermeable to top-down effects. This article reviews the original studies that (i) described the C1, (ii) linked it to primary visual cortex (V1) activity, and (iii) suggested that its electrophysiological characteristics are exclusively determined by lowlevel stimulus attributes, particularly the spatial position of the stimulus within the visual field. We then describe conflicting evidence from animal studies and human neuroimaging experiments and provide an overview of recent EEG and magnetoencephalography (MEG) work showing that initial V1 activity in humans may be strongly modulated by higher-level cognitive factors. Finally, we formulate a theoretical framework for understanding top-down effects on early visual processing in terms of predictive coding.
\end{abstract}

\title{
Keywords
}

attention; C1; ERPs; perceptual learning; predictive coding; primary visual cortex (V1) 


\section{Contents}

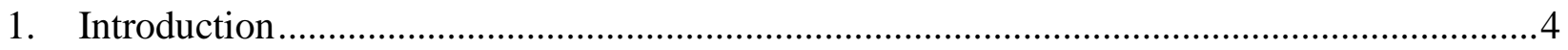

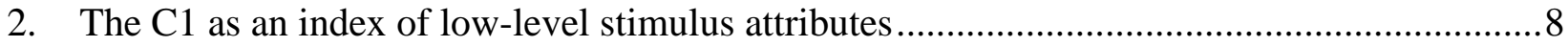

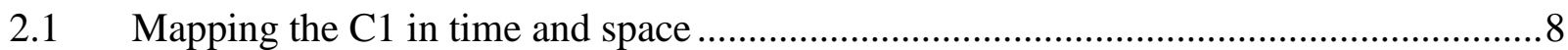

2.2 Absence of top-down effects ...................................................................... 13

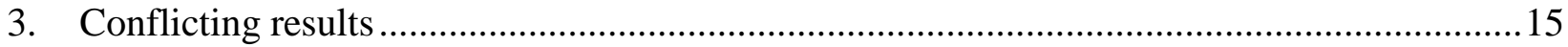

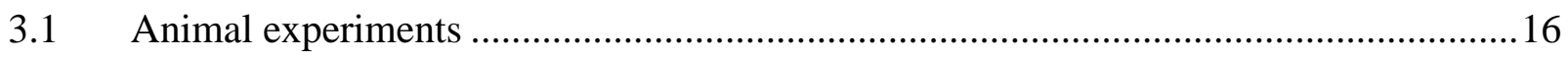

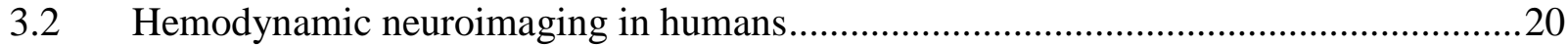

4. Recent evidence for top-down effects on early visual processing .....................................24

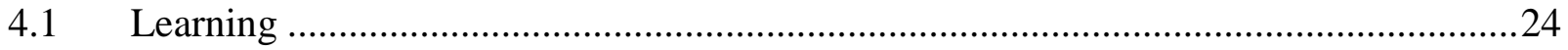

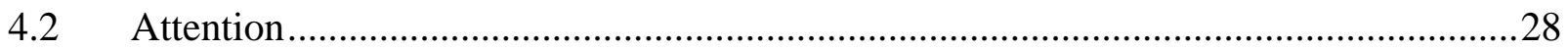

4.3 Prerequisites for observing top-down effects on early visual processing ...................33

5. A predictive coding framework for top-down effects on early visual processing .................35

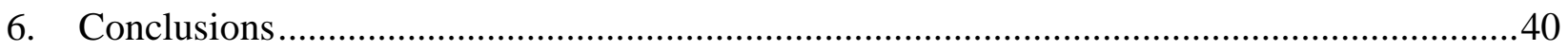




\section{Introduction}

In two experiments using two rows of electrodes, Jeffreys and Axford (1972a, b) described the characteristics of the earliest components of the visual evoked potential (VEP) in humans. As opposed to most studies before and since, they presented stimuli peripherally, rather than foveally or on the visual field meridians. In addition, these authors systematically tested for differences between stimuli restricted to different quadrants and octants, with a particular focus on variations between upper and lower visual fields. Based on their data (cf. also Jeffreys, 1971) and standard descriptions of the anatomy of the human visual system (Holmes, 1945), they concluded that the first of these components, characterized by an onset around $50 \mathrm{~ms}$ post-stimulus and peak latencies below $100 \mathrm{~ms}$, originates in V1.

Although it has since been demonstrated that frontal regions (e.g. the frontal eye-field, FEF) and extrastriate visual areas are already active at the peak latency of this earliest VEP component (Bullier, 2001; Foxe and Simpson, 2002; Thorpe et al., 1996), the basic assertion that what Jeffreys and Axford (1972a) termed "C. I" has its principal sources in primary visual cortex has stood the test of time (Clark et al., 1995; Di Russo et al., 2002; Fu et al., 2005; Fu et al., 2008; Im et al., 2006; Kelly et al., 2008; Martinez et al., 1999; Pourtois et al., 2004; Pourtois et al., 2008; Proverbio et al., 2010). The basic model proposed by Jeffreys and Axford (1972a) convincingly explains the most striking feature of the $\mathrm{C} 1$, which may also be the reason why the provisional name stuck: the $\mathrm{C} 1$ component reverses its polarity depending on whether the upper or lower visual field is stimulated, distinguishing it from later visual ERP components, such as the P1 or the N1. Hence, unlike these later deflections, the $\mathrm{C} 1$ corresponds to a retinotopic ERP component, as its amplitude is sensitive to the position of the stimulus in the visual field, whereas the P1 and N1 are influenced by the content of the stimulus or the task at hand, rather than stimulus location (Luck et al., 2000; Vogel and Luck, 2000). Because the standard nomenclature 
of numbered positive $(\mathrm{P})$ and negative $(\mathrm{N})$ peaks does not apply to this first visual component, some researchers have labeled it the NP80 (Bruin et al., 1998; Di Russo et al., 2005; Lange et al., 1998; Wijers et al., 1997). According to the Jeffreys and Axford model, the polarity reversal is due to the fact that V1 in the human principally covers the upper and lower banks of the calcarine sulcus, with the inversion between the external visual field and its central representation (Holmes, 1945) dictating that the upper visual field is represented on the lower bank of the calcarine and the lower visual field on the upper bank. Assuming a "canonical" calcarine sulcus running orthogonal to the interhemispheric fissure (Fig. 1A/B), selective stimulation of the upper or the lower visual field should activate populations of pyramidal neurons with opposing orientations, leading to the observed polarity reversal. At central posterior parietal electrodes, upper visual field stimulation then elicits a surface-negative component whereas the same stimulation in the lower visual field leads to a surface-positive potential characterized by the same distribution of the electric field (Fig. 1C; Clark et al., 1995).

\section{Insert Figure 1 around here}

The model depicted in Figure 1 explains why a $\mathrm{C} 1$ component is absent in many VEP studies. First, stimuli presented foveally activate regions situated close to the occipital pole (Fig. 1A) and the representation of the fovea shows large differences in terms of size and location between individuals (Dougherty et al., 2003), effectively smoothing out any clearly detectable VEP components at this very early latency. Hence, although a modest $\mathrm{C} 1$ component may still be detected for foveally presented stimuli (cf. Giard and Peronnet, 1999; Molholm et al., 2002), its amplitude is strongly reduced under these conditions, compared to when the same stimuli are presented at more eccentric locations in the periphery. Secondly, stimuli presented along the 
horizontal meridian tend to activate the fundus of the calcarine (Fig. 1B) and thus appear electrically neutral in EEG due to the tangential orientation with respect to the central parietooccipital electrodes (mostly Pz or POz) at which $\mathrm{C} 1$ is usually measured (Clark et al., 1995; Jeffreys and Axford, 1972a).

Several limitations need to be noted concerning this model, and Jeffreys and Axford (1972a) were well aware of them, describing it as "almost certainly an oversimplification for most subjects" (Jeffreys and Axford, 1972a, p. 18). First and foremost, individual differences in functional visual cortex anatomy are not limited to the representation of the fovea, but equally concern the location and extent of V1 and extrastriate visual areas, as well as the shape of the calcarine sulcus itself (Amunts et al., 2000; Dougherty et al., 2003; Hasnain et al., 1998). Accordingly, atypical C1 topographies may be observed (see Fig. 2), potentially making C1 measurements difficult to compare across subjects and studies (Kelly et al., 2008; Proverbio et al., 2007). A second, related caveat concerns the representation of the horizontal meridian, which in the majority of subjects does not seem to coincide exactly with the fundus of the calcarine, as its selective stimulation often evokes a negative C1 (Clark et al., 1995; Foxe et al., 2008). In many individuals, the representation of the lower visual field in fact extends across the fundus of the calcarine and into the lower bank of the sulcus. This asymmetry accords with known differences in the cortical representation of upper and lower visual fields in humans: higher contrast sensitivity and spatial resolution are usually observed in the lower visual field (Lehmann and Skrandies, 1979; Liu et al., 2006; Skrandies, 1987; Talgar and Carrasco, 2002), corresponding to a larger extent of the neuronal populations representing this part of the visual environment in primates (Van Essen et al., 1984).

\section{Insert Figure 2 around here}


Notwithstanding these limitations, the classical model as proposed by Jeffreys and Axford (1972a) still forms the basis of our current understanding of the C1 (Clark et al., 1995; Di Russo et al., 2002; Fu et al., 2008; Im et al., 2006; Kelly et al., 2008; Martinez et al., 1999; Pourtois et al., 2004; Pourtois et al., 2008; Vanni et al., 2004). As a potential indicator of very early activity in human primary visual cortex, the $\mathrm{C} 1$ has repeatedly been used to assess whether early stages of stimulus processing may be shaped by top-down factors, i.e. higher cognitive functions such as attention (e.g. Heinze et al., 1994). Until a few years ago, the overwhelming consensus emerging from these studies was that the $\mathrm{C} 1$ component is not affected by top-down control. More specifically, it was claimed that the well-characterized gain-control mechanisms of attention affecting later VEP components (Hillyard and Anllo-Vento, 1998; Hillyard et al., 1998b) do not act on this very early stage of visual cortex activity. Hence, while modulations of neural activity elicited by simple stimuli as a function of visuo-spatial selective attention have consistently been reported during early stages of sensory stimulus processing, they were typically found to take effect only after the offset of the $\mathrm{C} 1$ component. The fact that hemodynamic neuroimaging studies have repeatedly observed modulations of V1 activity due to selective attention (e.g. Martinez et al., 1999; Schwartz et al., 2005) was explained in terms of feedback effects. The latter are assumed to reflect modulations of neural activity due to recurrent processing loops, without affecting the initial volley of sensory input into V1 (Lee et al., 1998).

Recently, an increasing number of studies have cast doubts on the prevailing notion of $\mathrm{C} 1$ being impermeable to top-down control (Kelly et al., 2008; Poghosyan and Ioannides, 2008; Rauss et al., in press; Rauss et al., 2009). In the present article, we provide a detailed review of this ongoing discussion, linking it to similar disagreements in the animal literature and to a theoretical framework that may help explain divergent findings in the literature. In the following, 
we will first describe the relatively small number of studies that directly investigated the electrophysiological properties of the $\mathrm{C} 1$. We will then present an overview of experimental findings obtained from non-human primates and human hemodynamic neuroimaging studies challenging the view that early stages of processing in primary visual cortex are unaffected by higher cognitive processes. Finally, we will review recent EEG and MEG studies in humans showing that top-down modulations may actually influence the earliest VEP component, thus refuting a dogma that prevailed in the human electrophysiological literature over the last two decades.

\section{The $\mathrm{C} 1$ as an index of low-level stimulus attributes}

\subsection{Mapping the $C 1$ in time and space}

While polarity reversals between upper and lower visual field stimulation had been demonstrated before, the extensive mapping of half-fields, quadrants and octants as well as of different eccentricities allowed Jeffreys and Axford (1972a) to characterize the C1 in previously unmatched detail. Using tachistoscopically presented patterns of isolated squares and a transversal row of electrodes centered $2.5-5 \mathrm{~cm}$ anterior of the inion, they demonstrated (i) the characteristic polarity reversal of the $\mathrm{C} 1$ with upper vs. lower visual field stimulation; (ii) differences in $\mathrm{C} 1$ shape and distribution over the different electrodes following the stimulation of octants abutting the horizontal vs. vertical meridian, especially in the upper visual field; (iii) large individual differences in the degree of symmetry of $\mathrm{C} 1$ to unilateral stimulation in the left and right visual field; (iv) additivity of quadrant stimulation, such that the sum of activity elicited by

separately stimulating 2 adjacent quadrants is virtually equivalent to the corresponding half-field stimulation; (v) additivity of stimulation throughout the central $6^{\circ}$ of the visual field, with the 
largest contribution to the $\mathrm{C} 1$ stemming from the area between $2-6^{\circ}$. Based on these observations, the authors formulated the model described in the Introduction and tested it using dipole simulations.

The number of studies referring to Jeffreys and Axford (1972a) has remained moderate but constant, with 50 articles citing the seminal study in the 1970 s, 94 and 82 citations throughout the 1980 s and 90 s, respectively, and 73 citations in the past decade (source: ISI Web of Knowledge). Studies specifically investigating the C1 component, however, are substantially fewer and early results concerning the localization of its neural sources were not unequivocal. Specifically, a number of authors claimed that the $\mathrm{C} 1$ originated in extrastriate visual cortex (Lesevre and Joseph, 1979; Ossenblok and Spekreijse, 1991), whereas others (Butler et al., 1987) raised doubts about the model proposed by Jeffreys and Axford (1972a) because it does not make clear predictions about the responses elicited by foveal stimulation (for an overview, see Clark et al., 1995).

It was only after the seminal study by Clark et al. (1995) provided compelling evidence for $\mathrm{C} 1$ sources in primary visual cortex that cognitively oriented studies started to take the component into account (see the following sub-section). Clark et al. (1995) used high-density EEG to systematically map neural activity elicited by peripherally presented checkerboard patterns. Based on waveform, scalp current density (SCD; Perrin et al., 1989), and equivalent current dipole (ECD; Scherg and Berg, 1991) analyses, they largely confirmed the earlier findings of Jeffreys and co-workers (Jeffreys, 1971; Jeffreys and Axford, 1972a, b) and showed that the retinotopic and topographic characteristics of the $\mathrm{C} 1$ accord with its principal sources being located in primary visual cortex (Fig. 3).

Insert Figure 3 around here 
As the inverse problem of reconstructing neural sources from scalp EEG measurements allows for a theoretically unlimited number of solutions (Pasqual-Marqui et al., 1995; Scherg, 1990), subsequent ERP mapping studies improved the spatial localization of the C1 by adding high resolution anatomical data gathered using structural and functional magnetic resonance imaging (fMRI). For example, Di Russo and co-workers (Di Russo et al., 2002) presented circular checkerboard patterns in each quadrant while recording high-density EEG, with a subset of participants undergoing structural and functional MRI, the latter used to map retinotopically organized visual cortices (Sereno et al., 1995). They observed striate cortical generators only for the $\mathrm{C} 1$ component, whereas early and late phases of the $\mathrm{P} 1$ component were localized to dorsal and ventral extrastriate visual regions, respectively. The subsequent N1 complex (Vogel and Luck, 2000) was attributed to dipolar sources in regions overlapping those from which the P1 originated, as well as deep-lying sources in the parietal lobe. Importantly, the locations of dipolar sources for the $\mathrm{C} 1$ mapped reasonably well onto fMRI activations in early visual cortex obtained with the same stimuli.

Foxe and Simpson (2002) addressed the question of timing along the visual hierarchy. Using SCD analyses, they provided evidence for multiple-source activity during the $\mathrm{C} 1$ interval and very rapid propagation of activity especially along the dorsal visual stream (Bullier et al., 1996; Schall et al., 1995). Activity in frontal regions was observed as early as $30 \mathrm{~ms}$ after C1 onset, indicating that feedback may affect primary visual cortex activity within the first $100 \mathrm{~ms}$ post-stimulus and thus during the $\mathrm{C} 1$ interval. Based on these findings, Foxe and Simpson (2002) introduced the notion of an early $\mathrm{C} 1$ phase $\left(\mathrm{C}_{\mathrm{e}}\right)$, starting at around $50 \mathrm{~ms}$ post-stimulus and comprising the first 10-15 ms of the component, as the only part of the VEP that exclusively reflects V1 activity. 
Vanni and colleagues (Vanni et al., 2004) used uni- and bilateral checkerboard patterns in separate EEG and fMRI sessions and acquired additional retinotopic mapping data. Visual cortex activations in fMRI were then used as seeds for subsequent ECD source localizations. Stimuli were presented in the lower visual field and dipoles situated in V1 modeling the positive C1 could be clearly separated from sources in V3/V3A in most subjects. On the other hand, substantial crosstalk (measured in terms of similarity of location, orientation, and activation patterns of dipoles in different visual areas) was frequently present, such that sources in V2 could only rarely be distinguished from both V1 and V3/V3A sources. Similarly, for activity observed in V3/V3A during the rising phase of the $\mathrm{C} 1$, crosstalk from V1 could not definitely be excluded. Nevertheless, consistent with the findings of Foxe and Simpson (2002), Vanni et al. (2004) concluded that activity in extrastriate visual areas contributes to the generation of the $\mathrm{C} 1$. However, substantial delays of up to $20 \mathrm{~ms}$ were observed between successive peak source activity in areas $\mathrm{V} 1, \mathrm{~V} 2$, and $\mathrm{V} 3 / \mathrm{V} 3 \mathrm{~A}$, in accordance with the idea that the main source of activity during the $\mathrm{C} 1$ interval does have its origin in V1.

A comprehensive multimodal mapping study was published by Hagler et al. (2009), who used isoeccentric checkerboard patterns while simultaneously measuring EEG and MEG. In addition, they acquired structural and functional MRI, including retinotopic mapping data. Combining all modalities, the authors were able to substantially reduce the complexity of the inverse problem by mapping individual dipoles to the representation of each stimulus patch in early visual areas V1 through V3, effectively reducing cross-talk among the modeled sources in each individual. In accordance with the results of Foxe and Simpson (2002), relatively small delays were observed between sources reflecting activity in V1, V2, and V3, with substantial activation being present in extrastriate visual cortex at the peak latency of sources located in V1. Yet more recently, Ales et al. (2010a) proposed an even more sophisticated approach, with a 
large number of stimulus patches (96 or 192) used to map the 'folding fingerprint' of each individual's lower visual areas (V1 and V2 in their study). Their results are largely comparable to those reported by Hagler et al. (2009). Importantly, Ales et al. (2010a) observed similar response latencies in V1 and V2. They argued that polarity reversals such as that seen at the level of the $\mathrm{C} 1$, rather than reflecting pure $\mathrm{V} 1$ activity, only indicate that $\mathrm{V} 1$ activity contributes more than $50 \%$ of the component's variance. Although laborious (data from only 2 subjects were acquired in each of the two studies, and 500 min of fMRI scanning were required to achieve sufficient signal-to-noise ratio for the 196-patch stimulus used by Ales and co-workers), such multimodal mapping techniques appear highly promising to better map the loci of top-down modulatory effects on early sensory processing in space and time (see below).

Finally, Ales et al. (2010b) used forward modeling of EEG responses based on structurally and functionally mapped retinotopic visual areas in order to test whether polarity inversion of VEP components does contain information about the probable origin of such signals. Their results indicate that activity restricted to V1 should lead to polarity inversions for upper vs. lower visual field stimulation only in a minority of subjects, and that concurrent activity in areas V2 and V3 likely contributes to the polarity inversion of the $\mathrm{C} 1$. This presents a potentially serious challenge to the model proposed by Jeffreys and Axford (1972a), especially if the individual predictions of the forward model can be shown to match actual evoked-potential data. However, it does not invalidate the $\mathrm{C} 1$ as the earliest indicator of stimulus-evoked activity in retinotopic visual cortex. Thus, in the following, we may still refer to the $\mathrm{C} 1$ as a correlate of primary visual cortex activity, bearing in mind that extrastriate retinotopic areas are likely active during the same interval, but that the $\mathrm{C} 1$ remains the only reliable index of initial visual cortex activity using scalp-EEG in humans. 


\subsection{Absence of top-down effects}

Based on the description of the $\mathrm{C} 1$ as indexing the earliest response in primary visual cortex, several authors used the component as a tool to assess possible influences of cognitive factors on initial V1 activity triggered by a visual stimulus. Critically, the high temporal resolution of EEG (and MEG) offers a unique opportunity to assess distinct stages of visual processing over the duration of a couple of milliseconds after stimulus presentation, unlike other brain imaging methods such as positron-emission tomography (PET) or fMRI, which have a comparatively poor temporal resolution. A number of studies used the $\mathrm{C} 1$ to examine the influence of attention on early visual processing, with a particular focus on visuo-spatial selective attention. In this section, we will review studies that reported no effects of selective attention or other high-level processes on the amplitude or latency of the C1. Comprehensive overviews of the effects of attention on later ERP components have been published by Hillyard and co-workers (Hillyard and AnlloVento, 1998; Hillyard et al., 1998a; Hillyard et al., 1998b) and Mangun (1995).

In one of the earliest and most widely cited of these studies, Heinze and colleagues (1994) used concurrent PET and EEG to assess the effects of spatial attention on early visual responses. Subjects were instructed to pay attention to either the left or the right side of bilateral stimulus displays flashed into the upper visual field. Changes in regional cerebral blood-flow were observed in the fusiform gyrus and used as seeds for attentional effects on the P1. Despite the large differences in temporal resolution between the two methods, these seeded inverse solutions provided a good fit of the ERP data and relaxing the localization constraints produced very similar solutions. The authors specifically noted the absence of very early attentional effects, both at the level of the $\mathrm{C} 1$ component in the EEG data and at the level of V1 in PET.

Numerous EEG studies subsequently provided compelling evidence for the effects of spatial attention on early extrastriate VEP components (i.e., P1 and N1), but unequivocally 
reported no such effects earlier on, at the level of the $\mathrm{C} 1$. Experimental paradigms were similar to that employed by Heinze et al. (1994), with subjects covertly attending either the left or right visual field while uni- or bilateral stimulus displays were flashed into the periphery (Clark and Hillyard, 1996; Gomez Gonzalez et al., 1994; Johannes et al., 1995). Some experiments additionally examined the effects of color (Anllo-Vento and Hillyard, 1996; Lange et al., 1998) and background luminance (Wijers et al., 1997) to assess possible interactions between low-level stimulus characteristics and spatial attention, but also failed to find any attentional modulation of the $\mathrm{C} 1$ component.

Martinez et al. (1999) obtained fMRI (including retinotopic mapping) data and EEG recordings from the same subjects, using an experimental setup similar to Heinze et al. (1994), but with targets presented in a cluttered field of distractors. They found blood oxygen-level dependent (BOLD) signal changes as a function of attention throughout retinotopically organized visual areas, including V1. However, EEG recordings failed to show any evidence for C1 modulation by spatial attention (Fig. 4). Martinez et al. (1999) concluded that the effect of attention on V1 observed in the fMRI data reflects delayed feedback effects, rather than modulation of the initial sweep of activation.

\section{Insert Figure 4 around here}

A similar conclusion was reached by Noesselt et al. (2002), who simultaneously recorded EEG and MEG and obtained fMRI data in a separate recording session. Stimuli and procedure once more were similar to those employed by Heinze et al. (1994) and again, substantial effects of attention were evident in V1 from the fMRI data. However, neither MEG nor EEG recordings indicated any effects on the earliest component of the respective evoked responses. On the other 
hand, delayed attentional effects probably reflecting feedback connections to V1 were present both in the electric and the magnetic event-related responses, further supporting the notion of V1 being affected by top-down influences only beyond the earliest stage of stimulus-evoked activity.

The results of Martinez et al. (1999) and Noesselt et al. (2002) were followed up in an fMRI-informed EEG source localization study by Di Russo et al. (2003), who compared neural activity elicited by attended and unattended checkerboard stimuli in individual quadrants of the visual field. A single dipolar source in the vicinity of the calcarine sulcus was found to be unaffected by spatial attention during the $\mathrm{C} 1$ interval. However, the same source did show differential activity during a later time-window (150-225 ms), and this long-latency attentional modulation reversed polarity depending on whether the upper or lower visual field was stimulated. This symmetry between $\mathrm{C} 1$ activity and delayed attentional effects corroborated the notion that processing in the primary visual cortex may be shaped by attentional factors, but only at processing stages well beyond the initial part of the stimulus-evoked response.

In summary, these studies instituted the notion that V1 activity is modulated by higherorder influences such as spatial or feature-based attention; however, such modulations were only observed at relatively late, re-entrant processing stages, substantially later than the peak latency of the $\mathrm{C} 1$ and thus beyond the first sweep of activity passing through V1. In the following, we provide an overview of animal studies and human neuroimaging experiments that challenge this view.

\section{Conflicting results}




\subsection{Animal experiments}

A substantial body of evidence from animal studies supports the notion that the earliest sweep of activity in V1 may be subject to modulations by higher-order factors, including learning and attention. However, as described below, very early influences of higher cognitive processes on sensory processing are still debated in this field, as in humans. (Note that a comprehensive overview of the literature on early sensory processing in non-human primates and other species is beyond the scope of the present article; for a recent review, see Gilbert and Sigman, 2007).

Motter (1993) used a cued attention paradigm (Posner et al., 1980) to direct monkeys' attentional focus either toward or away from the receptive fields of V1, V2, and V4 neurons. Different numbers of stimuli were simultaneously presented and the author observed significant differences in firing rates as a function of focal attention in more than one-third of all neurons recorded in each visual area. Since firing rates differed between conditions from the very onset of neural responses, Motter (1993) suggested that preparatory effects during the cue-target interval must have affected neural responses to the target (see also Super et al., 2003). Importantly, attentional modulation in most neurons depended on the simultaneous presence of other stimuli in the display. This was the case even in V1 and although distances between stimuli were several times larger than the relevant receptive field diameters, thus excluding an explanation in terms of surround-suppression effects. Motter (1993) concluded that even the most basic levels of perception are subject to top-down effects, possibly mediated by local feedback connections within the visual system.

Gilbert and co-workers have conducted a series of studies demonstrating the active and dynamic characteristics of V1, a notion at odds with the previously held belief that it functions exclusively as a low-level feature detection module (for a detailed review, see Lee et al., 1998). Powerful modulations of V1 neurons' activity were induced by stimuli falling well outside their 
classical receptive field (Gilbert et al., 2000; Ito and Gilbert, 1999; Ito et al., 1998). Such effects are thought to reflect activity of local horizontal interconnections and suggest an important role for early visual cortex in contour integration and figure-background segregation (Roelfsema et al., 2007). Importantly, these contextual modulations were found to be subject to strong attentional influences, whereas the response to an individual target stimulus (i.e. without collinear flanking stimuli providing context information) remained virtually unchanged across different attentional conditions (Ito et al., 1998). In addition, extensive training not only led to perceptual learning in terms of decreased contrast thresholds, but also changed the nature of attentional effects on contextual information: In naïve monkeys, context effects were strong under conditions of distributed attention, where attention was allocated to several stimuli across the visual field; whereas focal attention following a cue that indicated the location of an upcoming target stimulus virtually abolished the benefits of collinear flankers. With training, however, performance under distributed attention approached the focused attention condition, demonstrating that attentional mechanisms themselves are subject to learning. The authors concluded that attention most likely acts as a gating mechanism at the level of long-range horizontal interconnections between V1 neurons of similar orientation preference. Moreover, they noted that attentional effects on contextual modulations led to increased firing rates from the very onset of the neural response (Ito and Gilbert, 1999).

In a later study, the same group (Crist et al., 2001) asked whether perceptual learning affects basic properties of V1 such as cortical magnification, the size of receptive fields, or orientation tuning specificity. All of these variables remained unchanged, whereas contextual modulations were strongly shaped by training. While animals performed either a fixation or a line-bisection task, the authors measured activity of V1 neurons with receptive fields not overlapping the task stimulus. They found that responses of these neurons to oriented bars placed 
inside the classical receptive field were modulated by flanking stimuli placed outside the classical receptive field, effectively creating a setup similar to the task-stimulus. Importantly, this modulation was found to be context-dependent: it occurred only if the animals were actively performing the experimental task, i.e. in a situation where the distance between two parallel bars was task-relevant. Since Crist et al. (2001) did not report data on the timing of the attentional modulations observed, their findings could be accounted for either by changes in early local V1 activity or alternatively by delayed feedback effects. However, Li and colleagues (Li et al., 2004) subsequently demonstrated similar effects in an experiment where the same physical stimuli were employed in two different task contexts (line bisection vs. Vernier tasks). Importantly, as in Ito and Gilbert (1999), the effects of task context were evident virtually from the first spike following stimulus presentation, providing strong evidence for the notion that top-down mechanisms can shape even the initial cortical stages of sensory processing.

By contrast, other studies have reported reliable modulations of V1 activity only at later stages of processing, challenging the idea that V1 may operate as a dynamic and flexible relay during early perception. For example, Roelfsema and others (Roelfsema et al., 1998) recorded from V1 in monkeys performing a curve-tracing task, which required the animals to distinguish between a target and a distracting stimulus. For spatially separated as well as intersecting curves, increased firing rates were observed along the whole extent of the target curve, but this objectbased attentional effect had an onset of > $200 \mathrm{~ms}$ and was thus substantially beyond the initial activity elicited by stimulus onset, which occurred with a latency of about $35 \mathrm{~ms}$. In accordance with the interpretations of Gilbert and colleagues (Gilbert et al., 2000; Ito and Gilbert, 1999; Ito et al., 1998), the authors concluded that long-range horizontal interconnections may be the neural substrate of the observed effects, with attention acting as a gate-keeping mechanism, although at longer latencies. 
Metha and colleagues (Mehta et al., 2000a, b) used an intermodal attention paradigm while simultaneously recording ERPs in multiple visual areas of the macaque with multi-contact electrodes spanning all layers of the cerebral cortex. Although they too observed attentional modulations of V1 activity, these were considerably weaker and occurred substantially later than those recorded in higher visual areas such as V4.

Using a figure-from-motion task, Roelfsema, Tolboom, and Khayat (2007) were able to distinguish different stages of visual processing in the alert monkey, namely feature detection, figure-background segregation, and attentional selection. They showed that each of these processing stages has a distinct neuronal correlate within V1, and that they are hierarchically organized: an early burst of activity due to motion onset was seen with a latency of $48 \mathrm{~ms}$; neuronal responses differed between figure and background as early as $57 \mathrm{~ms}$; and target stimuli elicited larger activity than distractors from $137 \mathrm{~ms}$ onward. This sequence of events, as well as its approximate timing, are in accordance with human scalp EEG results reviewed in the preceding section in that they suggest attentional modulations of V1 activity only as the result of re-entrant processing and substantially beyond the initial sweep of activation, as putatively reflected by the C1 (see Martinez et al., 1999; Noesselt et al., 2002)

In conclusion, the picture emerging from the animal literature is equivocal: very early modulations of sensory processing have been repeatedly observed in non-human primates (Ito and Gilbert, 1999; Ito et al., 1998; Li et al., 2004), whereas such effects were only detected at later processing stages by others (Mehta et al., 2000a; Roelfsema et al., 1998; Roelfsema et al., 2007). One reason for this discrepancy may lie in the different experimental paradigms used: it appears that early effects of attention were found whenever stimuli and task structure allowed for tonic changes of attentional parameters, such as the block-wise inhibition of one task-set in the experiments of $\mathrm{Li}$ et al. (2004), where some aspects of the visual stimulus were simply irrelevant 
during prolonged time intervals. On the other hand, if stimuli and task structure offer only a low level of predictability, where no part of the stimulus display can be tonically suppressed without the risk of reduced performance (Roelfsema et al., 1998; Roelfsema et al., 2007), attentional effects seem to occur at a later stage of processing in V1 and to involve recurrent processing in this region. In other words, attention may act as a gate-keeper at the level of bottom-up V1 activation only if this early gate in the processing sequence can be closed without substantial loss of information. Thus, while the continuous flow of incoming visual information is processed according to hierarchical principles following a gradual increase in the complexity of stimulus characteristics extracted in different perceptual modules, this flow of information may already be shaped at the earliest stage of processing within V1, depending on the activation of higher-level mental representations and whether they can be used to predict the temporal evolution or spatial distribution of upcoming visual stimuli.

The results of Mehta and co-workers (Mehta et al., 2000a, b), however, cannot be explained by this distinction and the question of when the earliest effects of top-down control may be observed in the non-human sensory cortex remains open to debate. Notwithstanding this caveat, the notion of V1 as an active and flexible processor (Gilbert and Sigman, 2007; Gilbert et al., 2001) is supported by these converging findings and is not in line with the previously dominant view of $\mathrm{V} 1$ as a simple and inflexible feature detection module. In the following, we will provide an overview of human neuroimaging studies that underline this relative flexibility of perceptual processing performed by V1.

\subsection{Hemodynamic neuroimaging in humans}

In humans, a large body of neuroimaging research has described top-down effects on metabolic and hemodynamic indicators of neural activity in early sensory cortices across a wide range of 
experimental paradigms. For example, Ress and co-workers (Ress et al., 2000) used a simple contrast-detection paradigm with auditory cueing to examine BOLD responses of retinotopically organized visual cortex as a function of stimulus presence vs. absence. They observed significant responses in V1 through V3 which were virtually the same whether a stimulus was presented or not. This 'base response' was systematically linked to behavioral performance, with larger base responses in all three areas predicting better performance (see also Mathewson et al., 2009; Super et al., 2003). Importantly, the base response was retinotopically specific in that it was observed only in visual cortex regions coding the experimental stimulus. Strikingly, these responses were absent at higher contrast levels, where behavioral performance was at ceiling. The authors interpreted trial-to-trial fluctuations in the base response as correlates of fluctuations of attention which in turn affect performance. Independent of its exact neurophysiological origins, the base response probably reflects a specific biasing signal recruited during difficult perceptual judgments and thus suggests the operation of top-down effects at the level of early visual cortex. However, as the authors pointed out themselves, it is impossible from their data to conclude whether the base response truly originated from V1 and was passed on to higher visual areas or vice versa.

We previously demonstrated that perceptual learning of a texture discrimination task (TDT; Karni and Sagi, 1991) selectively increases neural activity at the representation of the trained stimulus in V1 (Schwartz et al., 2002), and additional analyses of functional connectivity patterns suggested no involvement of other brain regions in mediating this effect following learning. In another study (Schwartz et al., 2005), increased attentional requirements of a task at central fixation were found to reduce the BOLD response elicited by irrelevant peripheral distractors even at the level of V1 (Fig. 5), with activity reductions conforming to a surroundsuppression profile (Smith, 2006). In both cases, long-range horizontal connections recruited 
during learning or gated as a function of attentional demands could conceivably mediate the suppression of task-irrelevant stimuli, in accordance with the proposals of Gilbert and co-workers (e.g. Gilbert and Sigman, 2007).

\section{Insert Figure 5 around here}

Using transcranial magnetic stimulation (TMS) concurrently with fMRI, Ruff et al. (2006) provided more direct evidence for top-down modulations from the frontal eye-field on early visual cortex activity. In their study, frontal eye-field TMS led to a characteristic pattern of increased BOLD responses throughout V1 to V4 for regions representing the peripheral visual field. Activity in retinotopic regions representing the central portion of the visual field, on the other hand, was reduced by TMS and this pattern of results was observed both in the presence and the absence of visual stimulation. Importantly, a separate psychophysical experiment showed behavioral effects in the same direction, i.e. contrast thresholds were decreased for peripherally presented stimuli (Gabor patches) during TMS. Thus, changes in frontal brain regions can modulate neuronal responses in early sensory cortices and these modulations can in turn modify the perception of simple stimuli (cf. Bullier, 2001; Thorpe et al., 1996).

In a continuous flash suppression paradigm, Bahrami et al. (2007) examined the effects of attentional load on the processing of invisible stimuli and observed reduced BOLD signal selectively in V1 under high load. Thus, even for stimuli that are unlikely to be processed beyond early visual areas, top-down modulations due to differing attentional demands affect hemodynamic responses in V1.

While these studies strongly suggest high-level influences on activity in V1, the delay of the hemodynamic response does not permit to draw strong conclusions about the time-course of 
the reported effects. However, numerous studies have also demonstrated effects of selective attention on pre-stimulus activity in early sensory cortices, suggesting that top-down mechanisms may exert relatively long-lasting influences on early sensory cortices and thereby influence the latter's response to subsequent stimulation (e.g. Kastner et al., 1999; Super et al., 2003).

Using spatial attention paradigms similar to those employed in numerous EEG studies, Gandhi et al. (1999) and Tootell et al. (1998) examined the effects of selective attention on V1 activity using fMRI. Both groups observed attentional modulations in V1 in the absence of visual stimuli, and Gandhi and colleagues reported that these modulations were on the order of $25 \%$ of stimulus-evoked activity.

Kastner and colleagues (Kastner et al., 1999) described distinct BOLD signal modulations corresponding to baseline shifts and attentional modulations of visually evoked responses in retinotopic visual areas. Both classes of effects differed as a function of simultaneous or sequential presentation of visual stimuli, as described previously by the same group (Kastner et al., 1998). Importantly, retinotopically specific increases of neural activity in preparation for peripherally presented stimuli was observed even at the level of V1, although this effect was less reliable than in higher visual areas (see also Schwartz et al., 2005).

Ruff and Driver (2006) showed that attentional modulations in visual cortex can be observed not only for target stimuli, but also for irrelevant distractors. In a psychophysical experiment, they found that advance knowledge reduced the behavioral costs associated with distractor occurrence. In a subsequent fMRI experiment, they observed changes in early visual cortex activity contralateral to cued distractors, and these changes were already present during the cue-target interval. These results suggest that top-down attentional influences on sensory processing may not only bias competition in favor of relevant stimuli, but may also serve to suppress neural processing of predictable distracting information, consistent with gain control 
exerted by attentional mechanisms onto lower-tier visual cortices (Hillyard et al., 1998b). However, since Ruff and Driver (2006) did not acquire retinotopic maps in their subjects, the specific involvement of V1 in these effects remains unclear.

In the following, we will turn to recent electrophysiological studies in humans whose results suggest that top-down effects on sensory processing may involve the earliest sweep of activation in primary visual cortex, as indexed by the $\mathrm{C} 1$.

\section{Recent evidence for top-down effects on early visual processing}

Over the last few years, an increasing number of EEG and MEG studies have provided evidence against the long-held dogma that V1 activity in humans is not reliably affected by top-down factors, but only by low-level physical properties including the position of the stimulus in the visual field. Below, we provide an exhaustive review of these studies in order to allow for a detailed assessment of why their results are different from those reviewed in Section 2.2. Note that while the $\mathrm{C} 1$ does not have a direct equivalent in MEG recordings, more lateralized components are usually detected during the $\mathrm{C} 1$ time-window (Chaumon et al., 2008) and these have been localized to V1 (Poghosyan and Ioannides, 2008).

\subsection{Learning}

We will first focus on recent studies suggesting long-term effects of basic learning mechanisms on early visual cortex activity. These provide evidence for the notion that the visual system remains plastic into adulthood and that flexibility in the early encoding of the visual stimulus can be detected using electrophysiological measures in humans. While learning is a multidimensional phenomenon that cannot be subsumed under the term 'top-down control', it usually implies some form of top-down modulation, for instance to monitor situations in which stored 
knowledge is to be applied. The study of $\mathrm{Li}$ et al. (2004) reviewed above is a particularly striking case in point: Following perceptual learning, a given V1 neuron can exhibit dramatically different activity patterns in response to the same physical stimulus, depending on current task demands. Thus, while the neural substrates of perceptual learning are likely located in early sensory cortices (Gilbert and Sigman, 2007), their application to a given environmental situation remains under top-down control. It is in this restricted sense that we discuss learning studies as potential evidence for top-down effects acting on early visual cortex activity.

Our group (Pourtois et al., 2008) previously demonstrated modulatory effects of perceptual learning on $\mathrm{C} 1$ amplitudes. In a direct follow-up of a previous fMRI study (Schwartz et al., 2002), we used the TDT (see above ; Karni and Sagi, 1991) to train participants' low-level orientation discrimination abilities selectively in one quadrant. Following a period of normal sleep necessary to stabilize learning effects on this and similar tasks (Stickgold and Walker, 2007), we measured EEG on the next day for stimuli presented in both trained and untrained visual quadrants. In order to limit the duration of the experiment and to avoid possible confounds by training several quadrants, two different groups of 14 subjects were trained and tested in either the lower or the upper visual field. Results indicated significant reductions of $\mathrm{C} 1$ amplitudes in the trained quadrant, but only for participants in the upper visual field group (Fig. 6). Differences during this early time-period were significant even when an integrative measure of electrical activity across the whole scalp (global field power, GFP, see Lehmann and Skrandies, 1980) was compared between trained and untrained conditions, indicating a reliable change in the strength of the ERP signal during this early time interval where the $\mathrm{C} 1$ was the highest. Furthermore, training did not affect subsequent P1 and N1 components, suggesting a component-specific effect of perceptual learning. The TDT primarily requires suppression of the distracting background texture, as shown by the fact that learning is specific to the orientation of background elements, 
but not target texture orientation (Karni and Sagi, 1991; Schwartz et al., 2002). Our results thus indicate that filtering mechanisms at the earliest levels of visual cortex activity can be trained effectively, and in turn influence the first sweep of cortical activation in response to trained stimuli. The fact that similar learning effects were not observed in the lower VF group may indicate retinotopic constraints on perceptual learning linked to known differences between upper and lower VF in terms of contrast sensitivity and spatial resolution (Carrasco et al., 2009; Lehmann and Skrandies, 1979; Rubin et al., 1996; Skrandies, 1987).

\section{Insert Figure 6 around here}

While perceptual learning is a relatively crude form of plasticity in the sense that it usually requires extensive training including hundreds to thousands of stimulus repetitions, more rapid forms of learning have also been shown to affect early visual cortex responses. Chaumon et al. (2008) used a modified contextual cueing paradigm and MEG to examine the effects of unconscious associative memory on the earliest stages of cortical visual processing. Repeatedly presented distractor patterns could be associated with the same target position in a visual search display, thus allowing predictions based on the distractors; in a non-predictive condition, on the other hand, target positions changed for each instance of a particular distractor pattern. Behavioral results suggested rapid learning effects, with mean reaction time differences of $80 \mathrm{~ms}$ for the last four of 12 presentations of each distractor pattern. Differences in MEG recordings between conditions were observed as early as 50-100 ms post-stimulus onset, selectively over posterior occipital scalp regions. Both this early time-course as well as the obtained topography suggest that these effects might be linked to differences in early visual cortex activity. Importantly, a battery of debriefing tests showed that participants remained unaware of the 
associations between the distractor patterns and target locations. Chaumon et al. (2008) concluded that early stages of processing in visual cortex may be influenced by implicitly learned contextual associations, possibly via an interaction of rapid feedback mechanisms and perceptual memories stored locally (see also Chaumon et al., 2009). Because previously acquired knowledge is used to gate early stimulus processing, these MEG results can be viewed as an instance of topdown control.

Interestingly, associative learning of emotional information has also been shown to affect early visual processing. Stolarova et al. (2006) examined emotional effects on early visual processing using an emotional conditioning paradigm. In their study, subjects underwent highdensity EEG recording during baseline, conditioning, and extinction of the association between peripherally presented gratings as conditioned stimuli (CSs) and centrally presented affective images as unconditioned stimuli (USs). It was found that CSs elicited significantly higher C1 amplitudes during conditioning blocks when compared to baseline or extinction periods. This extends findings of Pourtois et al. (2004), who observed significant C1 modulations for fearful as compared to neutral or happy faces (see also Halgren et al., 2000) used as emotional cues in a dot-probe task (Mogg et al., 1994). Importantly, C1 modulations were correlated with P1 effects on subsequent, non-emotional probe stimuli, suggesting a facilitatory effect of initial visual cortex responses to emotionally significant information on extrastriate processing of subsequent stimuli. Since behavioral performance was also increased for emotionally cued probe stimuli, these ERP results suggest that increased activity to emotional stimuli at the level of the $\mathrm{C} 1$ could serve an alerting function that translates into behavioral advantages. Alternatively, rapid feedback from subcortical structures involved in coarse emotional processing such as the amygdala (Morris et al., 1999; Vuilleumier, 2005) could have mediated emotional effects on both cue and probe processing. In combination with the results of Pourtois et al. (2004), the findings of 
Stolarova and colleagues (2006) suggest that emotional significance acquired on a relatively short time-scale can lead to differential gating of stimuli or plasticity-related changes at the earliest levels of processing in V1.

\subsection{Attention}

Having established that long-term changes due to associative or perceptual learning may modulate early V1 response characteristics, we now turn to recent studies suggesting similar modulations on shorter time-scales and related specifically to attentional task-demands.

Khoe et al. (2005) examined the effects of exogenous cueing in a transparent motion paradigm (Noest and van den Berg, 1993). In their experiment, two superimposed random-dot patterns rotated in opposite directions, creating the illusion of two transparent surfaces. A brief translation of one pattern was used to direct attention to one of the surfaces; a second translation could either occur for the cued or the uncued surface. Interestingly, the authors observed not only a modulation of the N1 component as found in previous studies using similar paradigms (Pinilla et al., 2001), but also increased $\mathrm{C} 1$ amplitudes if the second translation occurred for the previously cued surface. They suggested saturation effects on the $\mathrm{C} 1$ in previous studies as a possible reason for this novel result and argued that preferential treatment of the cued surface results from object-based attentional mechanisms. The effects of exogenous cueing on the $\mathrm{C} 1$ thus suggest short-term top-down effects on early activity in primary visual cortex.

Using advanced MEG source localization techniques, Poghosyan et al. (2005) observed modulation of V1 regions-of-interest as a function of attentional engagement vs. passive fixation of foveally presented stimuli within the first $100 \mathrm{~ms}$ following stimulus presentation. However, similar modulations were not observed for a smaller initial activation in $\mathrm{V} 1$ around 50 ms poststimulus. While the authors concluded that the initial feedforward activation of V1 remained 
unaffected by attention, their results suggest that feedback effects can act on V1 at substantially earlier latencies than previously believed on the basis of dipole modeling studies (e.g. Noesselt et al., 2002). However, due to the comparison of active task performance vs. passive fixation, it is difficult to rule out differences in arousal as a potential confounding factor.

Kelly and co-workers (Kelly et al., 2008) were among the first to publish ERP results indicating an effect of endogenous attention on $\mathrm{C} 1$ amplitudes. They covertly directed the attention of their participants to a particular location in the peripheral visual field using a centrally presented arrowhead and compared responses to peripherally presented gratings, which could be therefore attended or unattended, depending on the validity with the endogenous shift of spatial attention. Based on the findings and suggestions of Foxe and Simpson (2002), they used a separate mapping procedure to select in each subject the diametrically opposite locations in the upper and lower visual field associated with the largest difference between positive- and negative going $\mathrm{C} 1$ peaks. With this procedure taking into account the large individual differences in functional neuroanatomy of the visual cortex (see Section 1; Amunts et al., 2000; Dougherty et al., 2003; Hasnain et al., 1998) and the resulting variability of C1 topographies (Proverbio et al., 2007), they observed significant differences in early visual cortex activity as a function of endogenous attention. The subjects' task was to detect a slight change of contrast in the peripherally presented gratings and $\mathrm{C} 1$ amplitudes were found to be increased when subjects were actively attending to the location at which the grating appeared.

Interestingly, another recent study from the same group identified a confounding factor which may have obscured C1 effects in earlier experiments (Foxe et al., 2008), namely the spatial frequency content of visual stimuli. The authors designed their stimuli so as to activate either the magnocellular (M-) or the parvocellular (P-) system or both. The M-system has its origins in the M-ganglion cells of the retina, is sensitive to low contrasts, low spatial frequencies, and high 
temporal frequencies, and is believed to form the basis of the dorsal 'where'-pathway of the visual system. The P-system has its origins in the P-ganglion cells of the retina, responds only at higher contrast levels without saturating, possesses a low temporal but high spatial resolution, and is thought to form the basis of the ventral 'what'-pathway of the visual system (Kandel, 1991). Foxe et al. (2008) observed the highest C1 amplitudes for isoluminant chromatic contrast stimuli selectively targeting the P-pathway, intermediate C1 amplitudes in response to $100 \%$ contrast stimuli activating both P- and M-pathways, and a completely abolished $\mathrm{C} 1$ for $4 \%$ contrast stimuli selectively targeting the M-pathway.

Using MEG and a multisensory setup, Poghosyan and Ioannides (2008) observed attentional effects on early activity of auditory and visual primary cortices, both in their evoked potential data and in subsequent distributed source localization analyses. Specifically, spatial attention directed to one ear or one side of the visual field led to increased activity in regions implicated in simple sensory evoked activity, i.e. in the primary auditory and visual cortices, respectively. More importantly, the time-course of attentional effects coincided with stimulusdriven sensory activity without attention, arguing against the notion that primary sensory cortices are affected by top-down influences only at later, re-entrant stages of processing (Martinez et al., 1999; Roelfsema et al., 2007). Poghosyan and Ioannides (2008) also addressed the question of why previous studies using similar paradigms have overwhelmingly reported null findings. They suggest that source analyses using ECD approaches are ill-conceived for the study of early effects of attention in the visual domain, as they usually assume a single dipole at the level of V1, likely providing an oversimplified estimate of the local distribution of neural generators in this region which in turn does not agree with its well-established structural and anatomical landmarks (see Di Russo et al., 2002; Martinez et al., 1999). In light of the findings of Foxe and Simpson (2002) reviewed above, a single dipole contribution located in V1 seems indeed doubtful. Distributed 
source localization techniques could provide higher sensitivity for early attentional effects as they include simultaneously active sources, including regions that could act as the sources of putative top-down effects. This was empirically verified by additional ECD analyses, in which single sources used to fit the first component were either unconstrained or fixed to the locations obtained from the previous distributed source analyses. Results showed that the displacement of dipoles obtained in the unconstrained analyses effectively wiped out early attentional modulations of these sources. Regarding delayed attention effects as observed in animal experiments, Poghosyan and Ioannides (2008) argue that neural synchrony may play a central role in early attentional processes (Buehlmann and Deco, 2008; Engel et al., 2001; Fries et al., 2001) and that both single-unit activity and local current source density may not be sufficiently sensitive to large-scale synchrony to reliably detect attentional effects at very early processing stages.

Given these issues, it is interesting that in a recent EEG study, Karns and Knight (2009) report a dissociation between evoked gamma-band responses and ERPs for the tactile modality, where they observed early effects of intermodal attention only in the former. Importantly, they also extended the findings of Poghosyan and Ioannides (2008) by demonstrating very early effects of attention in both the auditory and the visual modality. In their task, subjects responded to stimuli in one modality, with the attended modality alternating between experimental blocks. Amplitudes of the $\mathrm{C} 1$ and N1 components as well as early evoked gamma-band responses were augmented when subjects were attending to visual stimuli. Attentional effects were restricted to the early phase of the $\mathrm{C}$, arguing against an extrastriate or re-entrant contribution (Foxe and Simpson, 2002).

Recently, we used an attentional load paradigm (Lavie, 1995; Lavie, 2005) to assess the effects of different degrees of attentional task engagement on the processing of peripheral 
distractors (Rauss et al., 2009). As in a previous fMRI experiment (Schwartz et al., 2005), participants judged either only the color or both the color and orientation of rapidly presented Tshapes at fixation while large, high-contrast distractors were unpredictably flashed in the periphery. Attentional load was varied between blocks and two groups of 14 subjects were tested in either the upper or the lower VF. Results demonstrated decreased C1 amplitudes under increased attentional load (Fig. 7), but this pattern was observed only for participants tested in the upper visual field. The findings were objectively verified using topographic microstate analyses (Michel et al., 1999; Pasqual-Marqui et al., 1995). They suggest that increased filtering of irrelevant distractors under heightened attentional demands is detectable at the earliest levels of visual cortex activity. Additional distributed source localization analyses using a local autoregressive procedure (LAURA; Grave de Peralta Menendez et al., 2004) showed load-related differences in dorsal and midline prefrontal structures which may constitute the sources of topdown effects on early visual processing stages (Corbetta and Shulman, 2002).

\section{Insert Figure 7 around here}

In a follow-up experiment (Rauss et al., in press), we changed the relative timing of central task stimuli and irrelevant peripheral stimuli by presenting them simultaneously. We observed significantly increased $\mathrm{C} 1$ amplitudes under high load and thus an inversion of the effect observed under non-simultaneous presentation conditions (Rauss et al., 2009). These results could suggest a dynamic interaction between endogenous attentional filtering and exogenous orienting mechanisms at the earliest stages of cortical visual processing. Alternatively, the effects may be explained in terms of a temporally focused recruitment of attention (Nobre et 
al., 2007) which would boost neural activity elicited by non-relevant stimuli that co-occur with the target, while suppressing such activity during the inter-target interval.

Finally, a recent study by Fu et al. (2009) reported interactive effects of exogenous attention and perceptual (as opposed to attentional) load on early visual processing. Nonpredictive cues were followed by target stimuli of differing levels of complexity. The authors observed a significant increase in $\mathrm{C} 1$ amplitudes for validly cued stimuli, selectively under high perceptual load. While the ensuing interaction between exogenous attention (cues) and perceptual load (targets) remained difficult to interpret in this study due to low-level stimulus differences, a follow-up study using stimuli that were balanced between load conditions replicated these findings (Fu et al., 2010a). An even more recent experiment by Fu and co-workers used the same paradigm to compare ERPs under active-task and passive-viewing conditions (Fu et al., 2010b). Finding no differences in $\mathrm{C} 1$ amplitudes, the authors concluded that attentional load does not affect early stages of processing in human visual cortex, in contrast to our findings (Rauss et al., in press; Rauss et al., 2009 ). It remains to be determined whether the comparison of active and passive viewing conditions engages the same attentional mechanisms as our manipulation of feature vs. conjunction detection.

\subsection{Prerequisites for observing top-down effects on early visual processing}

In summary, an increasing number of recent studies reporting effects of attention and learning on the $\mathrm{C} 1$ component and equivalent MEG indicators of early visual cortex activity suggests that the traditional view of V1 as a rigid stimulus-encoding module may be an oversimplification. Instead, provided that appropriate steps are taken to account for the properties of early visual cortex and its variability across subjects, significant top-down effects on the earliest stages of cortical visual processing in humans can be detected, revealing early modulatory effects of 
attention and learning. It is particularly interesting that both increases (Karns and Knight, 2009; Kelly et al., 2008; Khoe et al., 2005; Pourtois et al., 2004; Stolarova et al., 2006) and decreases (Pourtois et al., 2008; Rauss et al., 2009) of C1 amplitudes have been reported, in accordance with the stimulus characteristics and task demands of the different studies: thus, whereas the experiments from our group primarily assessed early filtering of irrelevant information and found reduced $\mathrm{C} 1$ amplitudes following learning or under increased attentional demands, others (e.g. Karns and Knight, 2009; Kelly et al., 2008) have shown increased C1 responses in tasks where increases in stimulus-evoked activity could conceivably aid task performance. The remarkable flexibility and adaptive tuning functions found in other primary sensory areas such as A1 (see Karns and Knight, 2009) thus seems to be equally present in V1. Based on these findings, the notion that V1 functions as an adaptive and dynamic processor (Gilbert and Sigman, 2007) is starting to gain support from the human EEG/MEG literature.

Possible reasons for the discrepancies between earlier studies which did not indicate higher-order influences on V1 activity in humans and the experiments just reviewed have already been alluded to and detailed discussions can be found in Kelly et al. (2008) and Pourtois et al. (2008). We will briefly summarize these issues and provide recommendations for an adequate assessment of top-down effects on early visual processing.

The combination of two factors seems to be principally responsible for previous failures to observe attentional or other high-level modulations at the level of the earliest components of the VEP recorded with either EEG or MEG. First, presentation conditions were usually not welladapted to the functional characteristics of V1. Thus, stimuli have frequently been presented on the horizontal meridian which may not be an ideal location for eliciting a clear $\mathrm{C} 1$ component in all subjects; if peripheral stimulus patterns were used, they were often small, activating only correspondingly small portions of V1, unlikely to produce equivalent EEG patterns in different 
subjects; conversely, task stimuli often extended well beyond the size of the small receptive fields in V1, effectively minimizing possible contributions of early visual cortex to task performance. In addition, differences in magno- and parvocellular contributions to the $\mathrm{C} 1$ as recently demonstrated by Foxe et al. (2008) indicate that stimuli have to be carefully designed in terms of spatial frequency and luminance contrast in order to adequately assess top-down effects on early visual processing.

Secondly, the large individual variability of early visual cortex observed in previous anatomical (Amunts et al., 2000) and functional (Dougherty et al., 2003; Hasnain et al., 1998) studies was usually not taken into account during either experimental design or data analyses. To our knowledge, the only studies to date directly addressing this issue are the previously reviewed experiments by Kelly et al. (2008), Hagler et al. (2009), and Ales et al. (2010a).

We suggest that future studies examining top-down effects on early visual cortex activity in humans more directly take into account at least one of these two fundamental issues: either experimental stimuli and tasks should be designed with a focus on whether the functional characteristics of V1 (i.e. small receptive fields, high degree of retinotopic organization, high contrast sensitivity) enable it to substantially contribute to task performance; or steps should be taken to appropriately consider individual variability in visual cortex anatomy. Ideally, both issued should be addressed at the same time. As indicated above, Hagler et al. (2009) and Ales et al. (2010a) have recently demonstrated that such a detailed assessment, although laborious, can be achieved with the current state of the art technology in human neuroimaging.

\section{A predictive coding framework for top-down effects on early visual processing}

The studies reviewed in the preceding section allow us to draw two conclusions: first, human V1 is not as inflexible and as impermeable to top-down modulation as was previously assumed; and 
second, these effects seem to be less pervasive than those observed for later VEP components. In particular, the detection of top-down modulations of the $\mathrm{C} 1$ seems to require highly specific experimental setups, such as individual mapping of the component's topography (Kelly et al., 2008), stimuli adapted to the characteristics of V1 (Foxe et al., 2008) including differences across the visual field (Pourtois et al., 2008), and close monitoring of stimulus timing (Rauss et al., in press; Rauss et al., 2009).

On the empirical level, recent findings showing very early modulations of sensory processing extend, rather than contradict, the large body of literature on the effects of attention on VEPs (Hillyard and Anllo-Vento, 1998; Hillyard et al., 1998a; Hillyard et al., 1998b). They show that under certain conditions, well-characterized gain control mechanisms of selective attention may already operate at the level of primary sensory cortices. On a conceptual level, however, the consequences of the emerging view of V1 as an adaptive processor (Gilbert and Sigman, 2007) are far-reaching. For example, it has been suggested that theories on the attentional functions of the thalamus (Crick, 1984; McAlonan et al., 2006) might be invalidated by experimental findings showing no influence of attention on initial processing in V1 (Heinze et al., 1994; Martinez et al., 1999). Yet, the evidence reviewed above suggests that there is no primary-secondary barrier separating V1 from higher-order visual areas in terms of attentional or other top-down influences. Rather, it is the functional characteristics of different cortical areas that determine the type and strength of top-down modulations that are observable at any particular level of processing within the context of a particular task.

While the neurophysiological implementation of such very early top-down effects on visual processing awaits further investigation, we believe that many of them can usefully be regarded as a consequence of predictive-coding mechanisms in the visual system. In section 3.1, we noted that relative predictability of a given stimulus or stimulus sequence may be one of the 
factors explaining divergent results in the animal literature. The notion that sensory systems form predictions of upcoming events is often neglected in experimental research (Summerfield and Egner, 2009). However, the functional properties of the visual system as a whole must somehow reflect the fact that, under natural viewing conditions, visual input changes constantly and predictably most of the time. Thus, low-level visual areas can be expected to adapt their processing characteristics in such a way as to preferentially retain or enhance representations of those aspects of a visual scene most likely to be affected by positive feedback (Serences, 2008); and to inhibit or filter representations that are least likely to be processed further. This notion is related to conceptualizations of visual cortex activity in terms of predictive coding, where neural signals are related less to a stimulus per se than to its congruence with internal goals and predictions, calculated on the basis of previous input to the system (Barlow, 1985; Mumford, 1992).

As reviewed by Rao and Ballard (1999), early studies in this direction were motivated mainly by the question of how the central nervous system achieves efficient (i.e. non-redundant) coding of perceptual input. Basic neurophysiological characteristics of V1 neurons such as endstopping have been successfully reproduced in predictive-coding models (Rao and Ballard, 1999; Spratling, 2010). More recently, fMRI studies in humans have shown that illusory contours elicit activity along the representation of equivalent real contours in early visual cortex (Muckli et al., 2005), and that stimuli appearing along an apparent-motion trajectory elicit less activity in V1 if they occur in synchrony with and in the same direction as the apparent motion stimulus (Alink et al., 2010). Instances of predictive coding have also been reported at the other end of cortical processing, with medial frontal regions encoding predictive templates against which perceptual input is presumably matched (Bar, 2004; Summerfield et al., 2006). Even supposedly low-level adaptation phenomena such as repetition suppression in fMRI have been re-interpreted in terms 
of predictive coding (Summerfield et al., 2008). Furthermore, a recent EEG experiment by Dambacher et al. (2009) showed that during sentence reading, visual ERPs differ within the first $100 \mathrm{~ms}$ post-stimulus between conditions in which predictable vs. unpredictable words were presented. This indicates a rapid comparison between expected and actual input to the visual language system involving the earliest stages of cortical visual processing, which is difficult to explain without assuming some sort of predictive coding-like mechanism. Finally, a study by Shuler and Bear (2006) indicates how low-level sensory cortices may attain predictive-coding properties: using implanted microelectrode arrays, they found that a substantial proportion of V1 neurons in the rat exhibit reward timing after a period of learning in a simple operant conditioning paradigm. Thus, these neurons coded stimulus-response contingencies rather than stimulus characteristics, reminiscent of the well-known activity patterns of dopaminergic midbrain neurons (Schultz et al., 1997).

Based on these studies and previous theoretical work (Bar, 2007; Gibson, 1986; Serences, 2008), we propose that the properties of early visual processing as assessed in the studies reviewed in this article reflect, not hard-wired properties of visual cortex, but contingencies learned by a flexible system in its accustomed visual environment. Learned contingencies in turn would be expressed in predictions about forthcoming sensory input. Thus, interpretation of recent findings indicating top-down modulations of early visual processing may be aided by considering which aspects of a given experimental task afford predictive coding and on what levels of the visual system such predictions would best be instantiated. One would expect that predictive coding can be readily employed only when there is an overlap between predictable environmental or task parameters and the functional characteristics of a given cortical area. In this sense, recent findings from our group may reflect differential applicability of predictive coding at the level of V1 in cases where irrelevant information is separated from relevant stimulus aspects both in 
space and time (Rauss et al., 2009), separated in space, but overlapping in time (Rauss et al., in press), or coinciding both in space and time (Pourtois et al., 2008).

As noted by Summerfield and Egner (2009), existing studies hardly allow for an estimation of the pervasiveness of predictive coding: perceptual expectations are rarely the focus of current research (for exceptions, see Doherty et al., 2005; Nobre et al., 2007), and experimental results interpreted in terms of selective attention are often confounded by expectation effects (e.g. Posner et al., 1980). Clarifying the relation between predictive coding and selective attention would seem of particular importance, as one of the most influential theories of selective attention, the biased-competition model (Desimone, 1998; Kastner et al., 1998; Luck et al., 1997), is usually assumed to contradict predictive-coding models of perception (Koch and Poggio, 1999). Although Spratling (2008a; 2008b) has shown that some computational implementations of the biased-competition model are mathematically equivalent to predictive coding, reconciling the empirical literature on the two topics will require considerable efforts (cf. Summerfield and Egner, 2009). Conceptually, the role of selective attention within a predictive-coding framework may relate to the fact that matching of higher-order predictions and incoming sensory data would have to be highly automatic and flexible at the same time under natural viewing conditions (cf. Mumford, 1992). We propose that attention could be one mechanism allowing for moment-to-moment flexibility in the relatively stable network of learned associations on which predictive coding is based. This flexibility could be achieved either by modulating the criteria for detecting a match between predictions and incoming information (i.e. by widening or narrowing the margin for signaling prediction errors), or by dynamically shifting the processing level at which violated predictions are regarded as behaviorally significant (Fig. 8). Testing these ideas and relating them to the existing literature may yield new perspectives on the interaction of perception, attention, and learning. 


\section{Insert Figure 8 around here}

In summary, we believe that future electrophysiological studies would benefit from explicitly considering to what extent predictive-coding mechanisms acting on early visual processing stages may contribute to task performance before concluding that top-down effects are observable at the level of V1 or not. The strongest possible test of our proposals would be to show that experimentally induced predictions affect the earliest stages of cortical visual processing even in the absence of visual stimulation. This would extend previous hemodynamic neuroimaging findings on preparatory effects in visual cortex (Chawla et al., 1999; Kastner et al., 1999; Ruff and Driver, 2006). Due to the often-cited advantages of electrophysiological methods in terms of temporal resolution, such studies could help determine whether preparatory activity can affect processing of subsequent stimuli very early along the visual hierarchy.

\section{Conclusions}

Recent evidence from human EEG and MEG studies suggests that the traditional view of V1 as an inflexible module for low-level stimulus encoding needs to be amended. Under appropriate conditions, effects of attention and learning on the earliest components of visually evoked electro- and magnetoencephalographic responses can be reliably detected. The notion that V1 acts as an adaptive and flexible processor, based mainly on animal studies, is thus gaining support from the human literature. We have proposed methodological improvements for future studies examining these issues, in order to better characterize the conditions under which the earliest stages of cortical visual processing may or may not be affected by top-down modulatory influences. These efforts should help create a framework for assessing the top-down permeability 
of early visual cortex, and may eventually help to determine whether very early effects of attention and learning on cortical processing play a role outside the artificial stimulus conditions created in the laboratory. We believe that consideration of the constraints and affordances of natural vision will lead to a conceptual enrichment of research into early visual processing, and that the notion of predictive coding, in particular, will help generate novel hypotheses allowing for a better characterization of the $\mathrm{C} 1$ component and, by extension, of the speed of visual cognition. 


\begin{abstract}
Abbreviations
BOLD, blood oxygen-level dependent; CS, conditioned stimulus; ECD, equivalent current dipole; EEG, electroencephalography; fMRI, functional magnetic resonance imaging; GFP, global field power; MEG, magnetoencephalography; PET, positron-emission tomography; primary visual cortex, V1; SCD, scalp current density; TMS, transcranial magnetic stimulation; US, unconditioned stimulus; VEP, visual evoked potential; VF, visual field
\end{abstract}

\title{
Acknowledgements
}

We would like to thank Patrik Vuilleumier and Christoph Michel as well as the whole Laboratory for Neurology and Imaging of Cognition and the Functional Brain Mapping Laboratory at the University of Geneva for valuable discussions. This work was supported by the Swiss National Science Foundation (grant \#310000-114008 to SS) and by a Geneva Neuroscience Center PhD fellowship (KR). GP is supported by grants from the European Research Council (Starting Grant \#200758) and Ghent University (BOF Grant \#05Z01708). 


\section{References}

Ales, J., Carney, T., Klein, S.A., 2010a. The folding fingerprint of visual cortex reveals the timing of human V1 and V2. NeuroImage 49, 2494-2502.

Ales, J.M., Yates, J.L., Norcia, A.M., 2010b. V1 is not uniquely identified by polarity reversals of responses to upper and lower visual field stimuli. NeuroImage 52, 1401-1409.

Alink, A., Schwiedrzik, C.M., Kohler, A., Singer, W., Muckli, L., 2010. Stimulus predictability reduces responses in primary visual cortex. J Neurosci 30, 2960-2966.

Amunts, K., Malikovic, A., Mohlberg, H., Schormann, T., Zilles, K., 2000. Brodmann's Areas 17 and 18 brought into stereotaxic space--where and how variable? NeuroImage 11, 66-84.

Anllo-Vento, L., Hillyard, S.A., 1996. Selective attention to the color and direction of moving stimuli: electrophysiological correlates of hierarchical feature selection. Percept Psychophys 58, 191-206.

Bahrami, B., Lavie, N., Rees, G., 2007. Attentional load modulates responses of human primary visual cortex to invisible stimuli. Curr Biol 17, 509-513.

Bar, M., 2004. Visual objects in context. Nat Rev Neurosci 5, 617-629.

Bar, M., 2007. The proactive brain: using analogies and associations to generate predictions. Trends Cogn Sci 11, 280-289.

Barlow, H.B., 1985. Cerebral cortex as model builder, in: Rose, D., Dobson, V.G. (Eds.), Models of the Visual Cortex. John Wiley \& Sons, Chichester, pp. 37-46.

Bruin, K.J., Kenemans, J.L., Verbaten, M.N., Van der Heijden, A.H., 1998. Localization of spatial attention processes with the aid of a probe technique. Electroencephalogr Clin Neurophysiol 108, 110-122.

Buehlmann, A., Deco, G., 2008. The neuronal basis of attention: rate versus synchronization modulation. J Neurosci 28, 7679-7686. 
Bullier, J., 2001. Integrated model of visual processing. Brain Res Rev 36, 96-107.

Bullier, J., Schall, J.D., Morel, A., 1996. Functional streams in occipito-frontal connections in the monkey. Behav Brain Res 76, 89-97.

Butler, S.R., Georgiou, G.A., Glass, A., Hancox, R.J., Hopper, J.M., Smith, K.R., 1987. Cortical generators of the CI component of the pattern-onset visual evoked potential.

Electroencephalogr Clin Neurophysiol 68, 256-267.

Carrasco, M., Eckstein, M., Verghese, P., Boynton, G., Treue, S., 2009. Visual attention: neurophysiology, psychophysics and cognitive neuroscience. Vision Res 49, 1033-1036.

Chaumon, M., Drouet, V., Tallon-Baudry, C., 2008. Unconscious associative memory affects visual processing before $100 \mathrm{~ms}$. J Vis 8, 1-10.

Chaumon, M., Schwartz, D., Tallon-Baudry, C., 2009. Unconscious learning versus visual perception: dissociable roles for gamma oscillations revealed in MEG. J Cogn Neurosci 21, 2287-2299.

Chawla, D., Rees, G., Friston, K.J., 1999. The physiological basis of attentional modulation in extrastriate visual areas. Nat Neurosci 2, 671-676.

Clark, V.P., Fan, S., Hillyard, S.A., 1995. Identification of early visual evoked potential generators by retinotopic and topographic analyses. Hum Brain Mapp 2, 170-187.

Clark, V.P., Hillyard, S.A., 1996. Spatial selective attention affects early extrastriate but not striate components of the visual evoked potential. J Cogn Neurosci 8, 387-402.

Corbetta, M., Shulman, G.L., 2002. Control of goal-directed and stimulus-driven attention in the brain. Nat Rev Neurosci 3, 201-215.

Crick, F., 1984. Function of the thalamic reticular complex: the searchlight hypothesis. Proc Natl Acad Sci U S A 81, 4586-4590. 
Crist, R.E., Li, W., Gilbert, C.D., 2001. Learning to see: experience and attention in primary visual cortex. Nat Neurosci 4, 519-525.

Dambacher, M., Rolfs, M., Göllner, K., Kliegl, R., Jacobs, A.M., 2009. Event-related potentials reveal rapid verification of predicted visual input. PLoS ONE 4, e5047.

Desimone, R., 1998. Visual attention mediated by biased competition in extrastriate visual cortex. Philos Trans R Soc Lond B Biol Sci 353, 1245-1255.

Di Russo, F., Martinez, A., Hillyard, S.A., 2003. Source Analysis of Event-related Cortical Activity during Visuo-spatial Attention. Cereb. Cortex 13, 486-499.

Di Russo, F., Martinez, A., Sereno, M.I., Pitzalis, S., Hillyard, S.A., 2002. Cortical sources of the early components of the visual evoked potential. Hum Brain Mapp 15, 95-111.

Di Russo, F., Pitzalis, S., Spitoni, G., Aprile, T., Patria, F., Spinelli, D., Hillyard, S.A., 2005. Identification of the neural sources of the pattern-reversal VEP. NeuroImage 24, 874-886. Doherty, J.R., Rao, A., Mesulam, M.M., Nobre, A.C., 2005. Synergistic effect of combined temporal and spatial expectations on visual attention. J Neurosci 25, 8259-8266.

Dougherty, R.F., Koch, V.M., Brewer, A.A., Fischer, B., Modersitzki, J., Wandell, B.A., 2003. Visual field representations and locations of visual areas V1/2/3 in human visual cortex. $\mathrm{J}$ Vis 3, 586-598.

Engel, A.K., Fries, P., Singer, W., 2001. Dynamic predictions: oscillations and synchrony in topdown processing. Nat Rev Neurosci 2, 704-716.

Foxe, J.J., Simpson, G.V., 2002. Flow of activation from V1 to frontal cortex in humans. A framework for defining "early" visual processing. Exp Brain Res 142, 139-150.

Foxe, J.J., Strugstad, E., Sehatpour, P., Molholm, S., Pasieka, W., Schroeder, C., McCourt, M., 2008. Parvocellular and magnocellular contributions to the initial generators of the visual 
evoked potential: high-density electrical mapping of the "C1" component. Brain Topogr 21, $11-21$

Fries, P., Reynolds, J.H., Rorie, A.E., Desimone, R., 2001. Modulation of oscillatory neuronal synchronization by selective visual attention. Science 291, 1560-1563.

Fu, S., Fedota, J., Greenwood, P.M., Parasuraman, R., 2010a. Early interaction between perceptual load and involuntary attention: An event-related potential study. Neurosci Lett $468,68-71$.

Fu, S., Fedota, J.R., Greenwood, P.M., Parasuraman, R., 2010b. Dissociation of visual C1 and P1 components as a function of attentional load: An event-related potential study. Biol Psychol $85,171-178$.

Fu, S., Greenwood, P.M., Parasuraman, R., 2005. Brain mechanisms of involuntary visuospatial attention: an event-related potential study. Hum Brain Mapp 25, 378-390.

Fu, S., Huang, Y., Luo, Y., Wang, Y., Fedota, J., Greenwood, P.M., Parasuraman, R., 2009. Perceptual load interacts with involuntary attention at early processing stages: event-related potential studies. NeuroImage 48, 191-199.

Fu, S., Zinni, M., Squire, P.N., Kumar, R., Caggiano, D.M., Parasuraman, R., 2008. When and where perceptual load interacts with voluntary visuospatial attention: An event-related potential and dipole modeling study. NeuroImage 39, 1345-1355.

Gandhi, S.P., Heeger, D.J., Boynton, G.M., 1999. Spatial attention affects brain activity in human primary visual cortex. Proc Natl Acad Sci U S A 96, 3314-3319.

Giard, M.H., Peronnet, F., 1999. Auditory-visual integration during multimodal object recognition in humans: a behavioral and electrophysiological study. J Cogn Neurosci 11, 473-490. 
Gibson, J.J., 1986. The Ecological Approach to Visual Perception. Lawrence Erlbaum Associates, Hillsdale, NJ, USA.

Gilbert, C.D., Ito, M., Kapadia, M., Westheimer, G., 2000. Interactions between attention, context and learning in primary visual cortex. Vision Res 40, 1217-1226.

Gilbert, C.D., Sigman, M., 2007. Brain states: top-down influences in sensory processing. Neuron 54, 677-696.

Gilbert, C.D., Sigman, M., Crist, R.E., 2001. The neural basis of perceptual learning. Neuron 31, 681-697.

Gomez Gonzalez, C.M., Clark, V.P., Fan, S., Luck, S.J., Hillyard, S.A., 1994. Sources of attention-sensitive visual event-related potentials. Brain Topogr 7, 41-51.

Grave de Peralta Menendez, R., Murray, M.M., Michel, C.M., Martuzzi, R., Gonzalez Andino, S.L., 2004. Electrical neuroimaging based on biophysical constraints. NeuroImage 21, $527-$ 539.

Hagler, D.J., Jr., Halgren, E., Martinez, A., Huang, M., Hillyard, S.A., Dale, A.M., 2009. Source estimates for MEG/EEG visual evoked responses constrained by multiple, retinotopicallymapped stimulus locations. Hum Brain Mapp 30, 1290-1309.

Halgren, E., Raij, T., Marinkovic, K., Jousmaki, V., Hari, R., 2000. Cognitive response profile of the human fusiform face area as determined by MEG. Cereb Cortex 10, 69-81.

Hasnain, M.K., Fox, P.T., Woldorff, M.G., 1998. Intersubject variability of functional areas in the human visual cortex. Hum Brain Mapp 6, 301-315.

Heinze, H.J., Mangun, G.R., Burchert, W., Hinrichs, H., Scholz, M., Munte, T.F., Gos, A., Scherg, M., Johannes, S., Hundeshagen, H., al, e., 1994. Combined spatial and temporal imaging of brain activity during visual selective attention in humans. Nature 372, 543-546. 
Hillyard, S.A., Anllo-Vento, L., 1998. Event-related brain potentials in the study of visual selective attention. Proc Natl Acad Sci U S A 95, 781-787.

Hillyard, S.A., Teder-Salejarvi, W.A., Munte, T.F., 1998a. Temporal dynamics of early perceptual processing. Curr Opin Neurobiol 8, 202-210.

Hillyard, S.A., Vogel, E.K., Luck, S.J., 1998b. Sensory gain control (amplification) as a mechanism of selective attention: electrophysiological and neuroimaging evidence. Philos Trans R Soc Lond B Biol Sci 353, 1257-1270

Holmes, G., 1945. The organization of the visual cortex in man. Proceedings of the Royal Society B $132,348-361$.

Im, C.H., Gururajan, A., Zhang, N., Chen, W., He, B., 2006. Spatial resolution of EEG cortical source imaging revealed by localization of retinotopic organization in human primary visual cortex. J Neurosci Methods.

Ito, M., Gilbert, C.D., 1999. Attention modulates contextual influences in the primary visual cortex of alert monkeys. Neuron 22, 593-604.

Ito, M., Westheimer, G., Gilbert, C.D., 1998. Attention and perceptual learning modulate contextual influences on visual perception. Neuron 20, 1191-1197.

Jeffreys, D.A., 1971. Cortical source locations of pattern-related visual evoked potentials recorded from the human scalp. Nature 229, 502-504.

Jeffreys, D.A., Axford, J.G., 1972a. Source locations of pattern-specific components of human visual evoked potentials. I. Component of striate cortical origin. Exp Brain Res 16, 1-21. Jeffreys, D.A., Axford, J.G., 1972b. Source locations of pattern-specific components of human visual evoked potentials. II. Component of extrastriate cortical origin. Exp Brain Res 16, $22-40$. 
Johannes, S., Münte, T.F., Heinze, H.J., Mangun, G.R., 1995. Luminance and spatial attention effects on early visual processing. Brain Res Cogn Brain Res 2, 189-205.

Kandel, E.R., 1991. Perception of motion, depth, and form, in: Kandel, E.R., Schwartz, J.H., Jessell, T.M. (Eds.), Principles of Neural Science, 3 ed. Elsevier, New York, pp. 440-466.

Karni, A., Sagi, D., 1991. Where practice makes perfect in texture discrimination: evidence for primary visual cortex plasticity. Proc Natl Acad Sci U S A 88, 4966-4970.

Karns, C.M., Knight, R.T., 2009. Intermodal auditory, visual, and tactile attention modulates early stages of neural processing. J Cogn Neurosci 21, 669-683.

Kastner, S., De Weerd, P., Desimone, R., Ungerleider, L.G., 1998. Mechanisms of directed attention in the human extrastriate cortex as revealed by functional MRI. Science 282, 108111.

Kastner, S., Pinsk, M.A., De Weerd, P., Desimone, R., Ungerleider, L.G., 1999. Increased activity in human visual cortex during directed attention in the absence of visual stimulation. Neuron 22, 751-761.

Kelly, S.P., Gomez-Ramirez, M., Foxe, J.J., 2008. Spatial attention modulates initial afferent activity in human primary visual cortex. Cereb Cortex 18, 2629-2636.

Khoe, W., Mitchell, J.F., Reynolds, J.H., Hillyard, S.A., 2005. Exogenous attentional selection of transparent superimposed surfaces modulates early event-related potentials. Vision Res 45, 3004-3014.

Koch, C., Poggio, T., 1999. Predicting the visual world: silence is golden. Nat Neurosci 2, 9-10. Lange, J.J., Wijers, A.A., Mulder, L.J., Mulder, G., 1998. Color selection and location selection in ERPs: differences, similarities and 'neural specificity'. Biol Psychol 48, 153-182.

Lavie, N., 1995. Perceptual load as a necessary condition for selective attention. J Exp Psychol Hum Percept Perform 21, 451-468. 
Lavie, N., 2005. Distracted and confused?: selective attention under load. Trends Cogn Sci 9, 7582.

Lee, T.S., Mumford, D., Romero, R., Lamme, V.A.F., 1998. The role of the primary visual cortex in higher level vision. Vision Res 38, 2429-2454.

Lehmann, D., Skrandies, W., 1979. Multichannel evoked potential fields show different properties of human upper and lower hemiretina systems. Exp Brain Res 35, 151-159.

Lehmann, D., Skrandies, W., 1980. Reference-free identification of components of checkerboardevoked multichannel potential fields. Electroencephalogr Clin Neurophysiol 48, 609-621.

Lesevre, N., Joseph, J.P., 1979. Modifications of the pattern-evoked potential (PEP) in relation to the stimulated part of the visual field (clues for the most probable origin of each component). Electroencephalogr Clin Neurophysiol 47, 183-203.

Li, W., Piech, V., Gilbert, C.D., 2004. Perceptual learning and top-down influences in primary visual cortex. Nat Neurosci 7, 651-657.

Liu, T., Heeger, D.J., Carrasco, M., 2006. Neural correlates of the visual vertical meridian asymmetry. J Vis 6, 1294-1306.

Luck, S.J., Chelazzi, L., Hillyard, S.A., Desimone, R., 1997. Neural Mechanisms of Spatial Selective Attention in Areas V1, V2, and V4 of Macaque Visual Cortex. J Neurophysiol 77, 24-42.

Luck, S.J., Woodman, G.F., Vogel, E.K., 2000. Event-related potential studies of attention. Trends Cogn Sci 4, 432-440.

Mangun, G.R., 1995. Neural mechanisms of visual selective attention. Psychophysiology 32, 418. 
Martinez, A., Anllo-Vento, L., Sereno, M.I., Frank, L.R., Buxton, R.B., Dubowitz, D.J., Wong, E.C., Hinrichs, H., Heinze, H.J., Hillyard, S.A., 1999. Involvement of striate and extrastriate visual cortical areas in spatial attention. Nat Neurosci 2, 364-369.

Mathewson, K.E., Gratton, G., Fabiani, M., Beck, D.M., Ro, T., 2009. To see or not to see: prestimulus \{alpha\} phase predicts visual awareness. J. Neurosci. 29, 2725-2732.

McAlonan, K., Cavanaugh, J., Wurtz, R.H., 2006. Attentional modulation of thalamic reticular neurons. J. Neurosci. 26, 4444-4450.

Mehta, A.D., Ulbert, I., Schroeder, C.E., 2000a. Intermodal selective attention in monkeys. I: distribution and timing of effects across visual areas. Cereb Cortex 10, 343-358.

Mehta, A.D., Ulbert, I., Schroeder, C.E., 2000b. Intermodal selective attention in monkeys. II: physiological mechanisms of modulation. Cereb Cortex 10, 359-370.

Michel, C.M., Seeck, M., Landis, T., 1999. Spatiotemporal dynamics of human cognition. News Physiol Sci 14, 206-214.

Mogg, K., Bradley, B.P., Hallowell, N., 1994. Attentional bias to threat: roles of trait anxiety, stressful events, and awareness. Q J Exp Psychol A 47, 841-864.

Molholm, S., Ritter, W., Murray, M.M., Javitt, D.C., Schroeder, C.E., Foxe, J.J., 2002. Multisensory auditory-visual interactions during early sensory processing in humans: a high-density electrical mapping study. Brain Res Cogn Brain Res 14, 115-128.

Morris, J.S., Ohman, A., Dolan, R.J., 1999. A subcortical pathway to the right amygdala mediating "unseen" fear. Proc Natl Acad Sci U S A 96, 1680-1685.

Motter, B.C., 1993. Focal attention produces spatially selective processing in visual cortical areas V1, V2, and V4 in the presence of competing stimuli. J Neurophysiol 70, 909-919.

Muckli, L., Kohler, A., Kriegeskorte, N., Singer, W., 2005. Primary visual cortex activity along the apparent-motion trace reflects illusory perception. PLoS Biol 3, e265. 
Mumford, D., 1992. On the computational architecture of the neocortex II. The role of corticocortical loops. Biol Cybern 66, 241-251.

Nobre, A.C., Correa, A., Coull, J.T., 2007. The hazards of time. Curr Opin Neurobiol 17, 465470.

Noesselt, T., Hillyard, S.A., Woldorff, M.G., Schoenfeld, A., Hagner, T., Jancke, L., Tempelmann, C., Hinrichs, H., Heinze, H.J., 2002. Delayed striate cortical activation during spatial attention. Neuron 35, 575-587.

Noest, A.J., van den Berg, A.V., 1993. The role of early mechanisms in motion transparency and coherence. Spat Vis 7, 125-147.

Ossenblok, P., Spekreijse, H., 1991. The extrastriate generators of the EP to checkerboard onset. A source localization approach. Electroencephalogr Clin Neurophysiol 80, 181-193.

Pasqual-Marqui, R.D., Michel, C.M., Lehmann, D., 1995. Segmentation of brain electrical activity into microstates: model estimation and validation. IEEE Trans Biomed Eng 42, $658-665$.

Perrin, F., Pernier, J., Bertrand, O., Echallier, J.F., 1989. Spherical splines for scalp potential and current density mapping. Electroencephalogr Clin Neurophysiol 72, 184-187.

Pinilla, T., Cobo, A., Torres, K., Valdes-Sosa, M., 2001. Attentional shifts between surfaces: effects on detection and early brain potentials. Vision Res 41, 1619-1630.

Poghosyan, V., Ioannides, A.A., 2008. Attention Modulates Earliest Responses in the Primary Auditory and Visual Cortices. Neuron 58, 802-813.

Poghosyan, V., Shibata, T., Ioannides, A.A., 2005. Effects of attention and arousal on early responses in striate cortex. Eur J Neurosci 22, 225-234.

Posner, M., Snyder, C., Davidson, B., 1980. Attention and the detection of signals. J Exp Psychol $109,160-174$. 
Pourtois, G., Grandjean, D., Sander, D., Vuilleumier, P., 2004. Electrophysiological correlates of rapid spatial orienting towards fearful faces. Cereb Cortex 14, 619-633.

Pourtois, G., Rauss, K.S., Vuilleumier, P., Schwartz, S., 2008. Effects of perceptual learning on primary visual cortex activity in humans. Vision Res 48, 55-62.

Proverbio, A.M., Del Zotto, M., Zani, A., 2007. Inter-individual differences in the polarity of early visual responses and attention effects. Neurosci Lett 419, 131-136.

Proverbio, A.M., Del Zotto, M., Zani, A., 2010. Electrical neuroimaging evidence that spatial frequency-based selective attention affects V1 activity as early as 40-60 ms in humans. BMC Neurosci 11, 59.

Rao, R.P.N., Ballard, D.H., 1999. Predictive coding in the visual cortex: a functional interpretation of some extra-classical receptive-field effects. Nat Neurosci 2, 79-87.

Rauss, K., Pourtois, G., Vuilleumier, P., Schwartz, S., in press. Effects of attentional load on early visual processing depend on stimulus timing. Hum Brain Mapp.

Rauss, K.S., Pourtois, G., Vuilleumier, P., Schwartz, S., 2009. Attentional load modifies early activity in human primary visual cortex. Hum Brain Mapp 30, 1723-1733.

Ress, D., Backus, B.T., Heeger, D.J., 2000. Activity in primary visual cortex predicts performance in a visual detection task. Nat Neurosci 3, 940-945.

Roelfsema, P.R., Lamme, V.A., Spekreijse, H., 1998. Object-based attention in the primary visual cortex of the macaque monkey. Nature 395, 376-381.

Roelfsema, P.R., Tolboom, M., Khayat, P.S., 2007. Different processing phases for features, figures, and selective attention in the primary visual cortex. Neuron 56, 785-792.

Rubin, N., Nakayama, K., Shapley, R., 1996. Enhanced perception of illusory contours in the lower versus upper visual hemifields. Science 271, 651-653. 
Ruff, C.C., Blankenburg, F., Bjoertomt, O., Bestmann, S., Freeman, E., Haynes, J.D., Rees, G., Josephs, O., Deichmann, R., Driver, J., 2006. Concurrent TMS-fMRI and psychophysics reveal frontal influences on human retinotopic visual cortex. Curr Biol 16, 1479-1488.

Ruff, C.C., Driver, J., 2006. Attentional preparation for a lateralized visual distractor: behavioral and fMRI evidence. J Cogn Neurosci 18, 522-538.

Schall, J.D., Morel, A., King, D.J., Bullier, J., 1995. Topography of visual cortex connections with frontal eye field in macaque: convergence and segregation of processing streams. $\mathbf{J}$ Neurosci 15, 4464-4487.

Scherg, M., 1990. Fundamentals of dipole source potential analysis, in: Grandori, F., Hoke, M., Romani, G.L. (Eds.), Auditory Evoked Magnetic Fields and Electric Potentials. Karger, Basel, pp. 40-69.

Scherg, M., Berg, P., 1991. Use of prior knowledge in brain electromagnetic source analysis. Brain Topogr 4, 143-150.

Schultz, W., Dayan, P., Montague, P.R., 1997. A neural substrate of prediction and reward. Science 275, 1593-1599.

Schwartz, S., Maquet, P., Frith, C., 2002. Neural correlates of perceptual learning: a functional MRI study of visual texture discrimination. Proc Natl Acad Sci U S A 99, 17137-17142.

Schwartz, S., Vuilleumier, P., Hutton, C., Maravita, A., Dolan, R.J., Driver, J., 2005. Attentional load and sensory competition in human vision: modulation of fMRI responses by load at fixation during task-irrelevant stimulation in the peripheral visual field. Cereb Cortex 15, 770-786.

Serences, J.T., 2008. Value-based modulations in human visual cortex. Neuron 60, 1169-1181. 
Sereno, M.I., Dale, A.M., Reppas, J.B., Kwong, K.K., Belliveau, J.W., Brady, T.J., Rosen, B.R., Tootell, R.B., 1995. Borders of multiple visual areas in humans revealed by functional magnetic resonance imaging. Science 268, 889-893.

Shuler, M.G., Bear, M.F., 2006. Reward timing in the primary visual cortex. Science 311, 16061609.

Skrandies, W., 1987. The upper and lower visual field of man: electrophysiological and functional differences, in: Ottoson, D. (Ed.), Progress in Sensory Physiology. Springer, Berlin.

Smith, M.A., 2006. Surround suppression in the early visual system. J Neurosci 26, 3624-3625. Spratling, M.W., 2008a. Predictive coding as a model of biased competition in visual attention. Vision Res 48, 1391-1408.

Spratling, M.W., 2008b. Reconciling predictive coding and biased competition models of cortical function. Frontiers Computat Neurosci 2.

Spratling, M.W., 2010. Predictive coding as a model of response properties in cortical area V1. J Neurosci 30, 3531-3543.

Stickgold, R., Walker, M.P., 2007. Sleep-dependent memory consolidation and reconsolidation. Sleep Med 8, 331-343.

Stolarova, M., Keil, A., Moratti, S., 2006. Modulation of the C1 visual event-related component by conditioned stimuli: evidence for sensory plasticity in early affective perception. Cereb Cortex 16, 876-887.

Summerfield, C., Egner, T., 2009. Expectation (and attention) in visual cognition. Trends Cogn Sci 13, 403-409.

Summerfield, C., Egner, T., Greene, M., Koechlin, E., Mangels, J., Hirsch, J., 2006. Predictive codes for forthcoming perception in the frontal cortex. Science 314, 1311-1314. 
Summerfield, C., Trittschuh, E.H., Monti, J.M., Mesulam, M.M., Egner, T., 2008. Neural repetition suppression reflects fulfilled perceptual expectations. Nat Neurosci 11, 10041006.

Super, H., van der Togt, C., Spekreijse, H., Lamme, V.A.F., 2003. Internal state of monkey primary visual cortex (V1) predicts figure-ground perception. Journal of Neuroscience 23, 3407-3414.

Talgar, C.P., Carrasco, M., 2002. Vertical meridian asymmetry in spatial resolution: visual and attentional factors. Psychon Bull Rev 9, 714-722.

Thorpe, S., Fize, D., Marlot, C., 1996. Speed of processing in the human visual system. Nature $381,520-522$.

Tootell, R.B., Hadjikhani, N., Hall, E.K., Marrett, S., Vanduffel, W., Vaughan, J.T., Dale, A.M., 1998. The retinotopy of visual spatial attention. Neuron 21, 1409-1422.

Van Essen, D.C., Newsome, W.T., Maunsell, J.H., 1984. The visual field representation in striate cortex of the macaque monkey: asymmetries, anisotropies, and individual variability. Vision Res 24, 429-448.

Vanni, S., Warnking, J., Dojat, M., Delon-Martin, C., Bullier, J., Segebarth, C., 2004. Sequence of pattern onset responses in the human visual areas: an fMRI constrained VEP source analysis. NeuroImage 21, 801-817.

Vogel, E.K., Luck, S.J., 2000. The visual N1 component as an index of a discrimination process. Psychophysiol 37, 190-203.

Vuilleumier, P., 2005. How brains beware: neural mechanisms of emotional attention. Trends Cogn Sci 9, 585-594. 
Wijers, A.A., Lange, J.J., Mulder, G., Mulder, L.J., 1997. An ERP study of visual spatial attention and letter target detection for isoluminant and nonisoluminant stimuli. Psychophysiol 34, 553-565. 


\section{Figure Captions}

Figure 1. Basic model explaining the characteristic polarity reversal of the $\mathrm{C} 1$. (A) The retinotopic representation of the visual environment in V1 contains central locations ('c') at the posterior end of the calcarine sulcus and more peripheral locations (' $p$ ') at more anterior sites within the sulcus. (B) Structural MRI with the calcarine sulcus highlighted in red on sagittal and coronal views (the plane of the coronal slice is marked in the sagittal view). The fundus of the calcarine (' $\mathrm{f}$ ') roughly corresponds to the representation of the horizontal meridian in V1. (C) Populations of neurons of opposite orientation in the calcarine sulcus are activated by upper vs. lower visual field stimulation. V1 is the only early visual area where neurons coding for these different parts of the visual field are consistently oriented in opposite directions. The early onset $(\sim 50 \mathrm{~ms})$ and peak latency $(<100 \mathrm{~ms})$ of the $\mathrm{C} 1$ additionally suggest neural generators in lowerlevel visual areas.

Figure 2. Grand-average and single subject ERPs from a perceptual learning experiment (Pourtois et al., 2008). Top: Traces show VEPs at electrode Pz for a group of 12 subjects (blue trace) and for an individual subject showing a highly atpyical $\mathrm{C} 1$ topography (red trace). Bottom:

Topographic maps at the time of the $\mathrm{C} 1$ peak (90 ms post-stimulus) for the grand average (left) and the individual subject shown above (right). Maps are scaled to $\pm 7.5 \mu \mathrm{V}$. The subject in question was excluded from analysis. While extreme cases such as this one are relatively rare, the component's distribution shows considerable variation between subjects, in accordance with the known variability of visual cortex anatomy in humans. Such variability includes differences in 
the angle between the calcarine sulcus and the interhemishperic fissure that which could explain bimodal $\mathrm{C} 1$ distributions as seen in this subject.

Figure 3. Results of Clark et al. (1995). The image shows topographic maps of the C1 and emerging P1 component for checkerboards presented at different locations in the visual field. It illustrates the polarity reversal of the $\mathrm{C} 1$ for upper vs. lower field stimulation, as well as the persistence of a negative $\mathrm{C} 1$ for stimuli located slightly below the horizontal meridian. The inset shows the schematic model that Clark and colleagues formulated on the basis of dipole simulations performed on their data. This model corroborates and specifies the original proposals of Jeffreys and Axford (1972a).

Figure 4. Results of Martinez et al. (1999). Subjects attended either to the left or to the right of the stimulus display, leading to large differences at the level of the P1 and N1 components elicited by the same physical stimuli. However, no differences were found at the level of the $\mathrm{C} 1$ (A). In contrast to the ERP findings, overlays of attentional effects onto individually mapped retinotopic cortices showed clear differences in primary visual cortex (2). Red overlays indicate higher activity when attention was directed to the left visual field; blue overlays show increased activity in the attend-right condition.

Figure 5. Attentional load reduces V1 response to irrelevant peripheral stimuli. (A) Visual cortex responses to peripherally presented checkerboard patterns were compared between conditions of low and high attentional load imposed by the task at fixation. (B) Data from four representative subjects. Retinotopically mapped visual areas are overlaid in different colors on individual hemispheres. White overlays indicate regions of decreased visual cortex responses under high 
attentional load, reflecting stronger filtering of irrelevant information under higher attentional demands. Adapted from Schwartz et al. (2005)

Figure 6. Perceptual learning reduces $\mathrm{C} 1$ amplitudes. (A) Procedure of the texture discrimination task. Participants were trained to identify an arrangement of diagonal bars hidden in an array of horizontal bars. Training was restricted to one quadrant of the visual field, whereas testing on the following day was performed in both the trained and the untrained quadrant in either the upper or the lower visual field. (B) Grand-average ERPs over nine posterior electrodes located around the midline. A significant reduction of $\mathrm{C} 1$ amplitudes was observed following training, but this effect was found only in the upper visual field. Adapted from Pourtois et al. (2008)

Figure 7. Attentional load affects $\mathrm{C} 1$ amplitudes elicited by irrelevant peripheral stimuli. (A) Experimental setup. The task was the same as in Schwartz et al. (2005) shown in Fig. 5. Irrelevant arrays of horizontal bars were flashed randomly in the periphery, to probe V1 reactivity during blocks of low vs. high attentional load. (B) Grand-average topographies and ERP waveshapes for participants tested in the upper and lower visual field. Significant C1 reductions were observed under high attentional load in the upper visual field group. Adapted from Rauss et al. (2009).

Figure 8. Predictive coding in visual perception (adapted from Summerfield and Egner, 2009; based on Rao and Ballard, 1999; Spratling, 2008). Higher cortical areas such as prefrontal cortex (PFC) transmit predictions of upcoming visual stimuli onto representation units $(\mathrm{R})$ in lower areas. $\mathrm{R}$ units encode probability distributions for each stimulus along several stimulus dimensions (only one shown for simplicity). Sensory input is compared to predictive templates 
via interactions between $\mathrm{R}$ units and error $(\mathrm{E})$ units. The latter carry prediction error $(\mathrm{PE})$ signals to higher areas, either in visual cortex (e.g. V4) or outside (e.g. PFC). The example assumes two stimuli that can be distinguished at the level of V4 (e.g. red and green gratings of different orientiations). V4 sends predictive templates concerning characteristics stimulus elements (e.g. orientation) to V1. Both the initiation of templates and the template-matching process are highly automatic. Selective attention may affect these automatic processes by adjusting the margins within which a match between sensory input and a particular template is detected. In addition, attention may enhance or reduce the effects of prediction errors signalled by each module if advance knowledge suggests that a module‘s output is particularly important or completely irrelevant. Based on current knowledge about top-down modulations of early visual cortex activity, it is proposed that selective attention and other high-level processes cannot easily modulate predictive templates stored in V1, but that they may act by changing the weighting of that output. This could occur either by reducing the effect of PE signalling on higher levels of the hierarchy, or by adapting the threshold for PE signals to be passed on. 

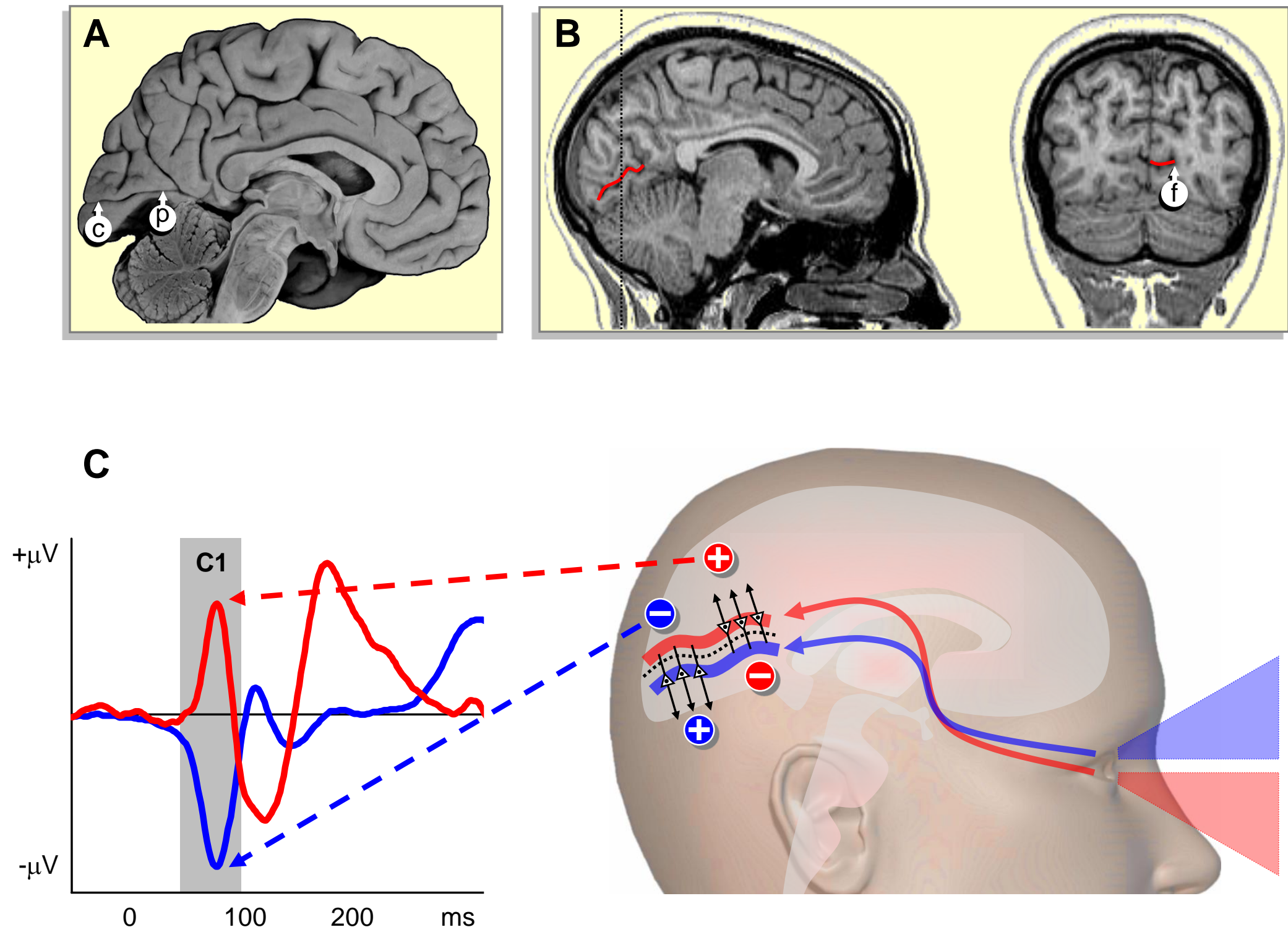


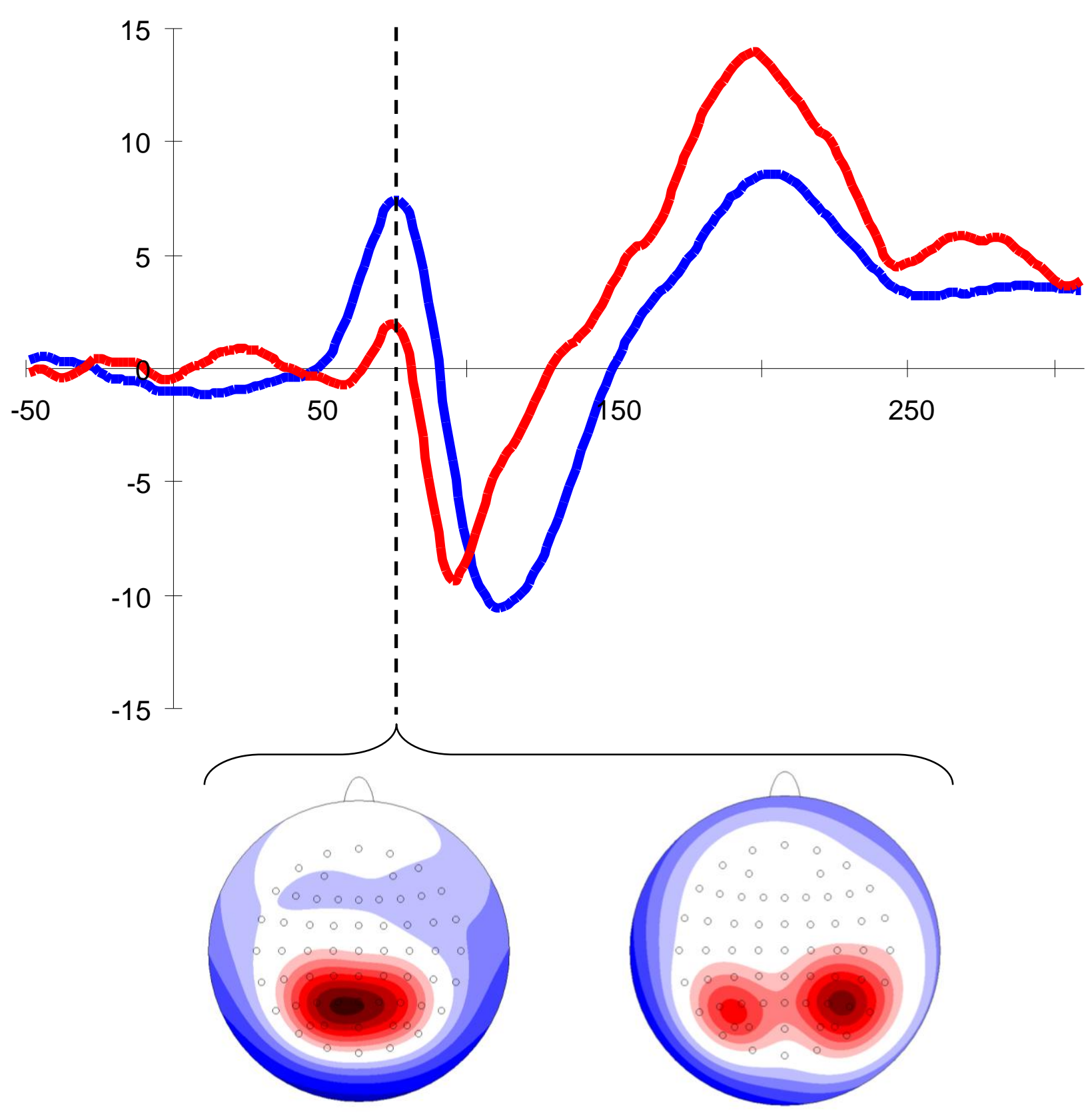




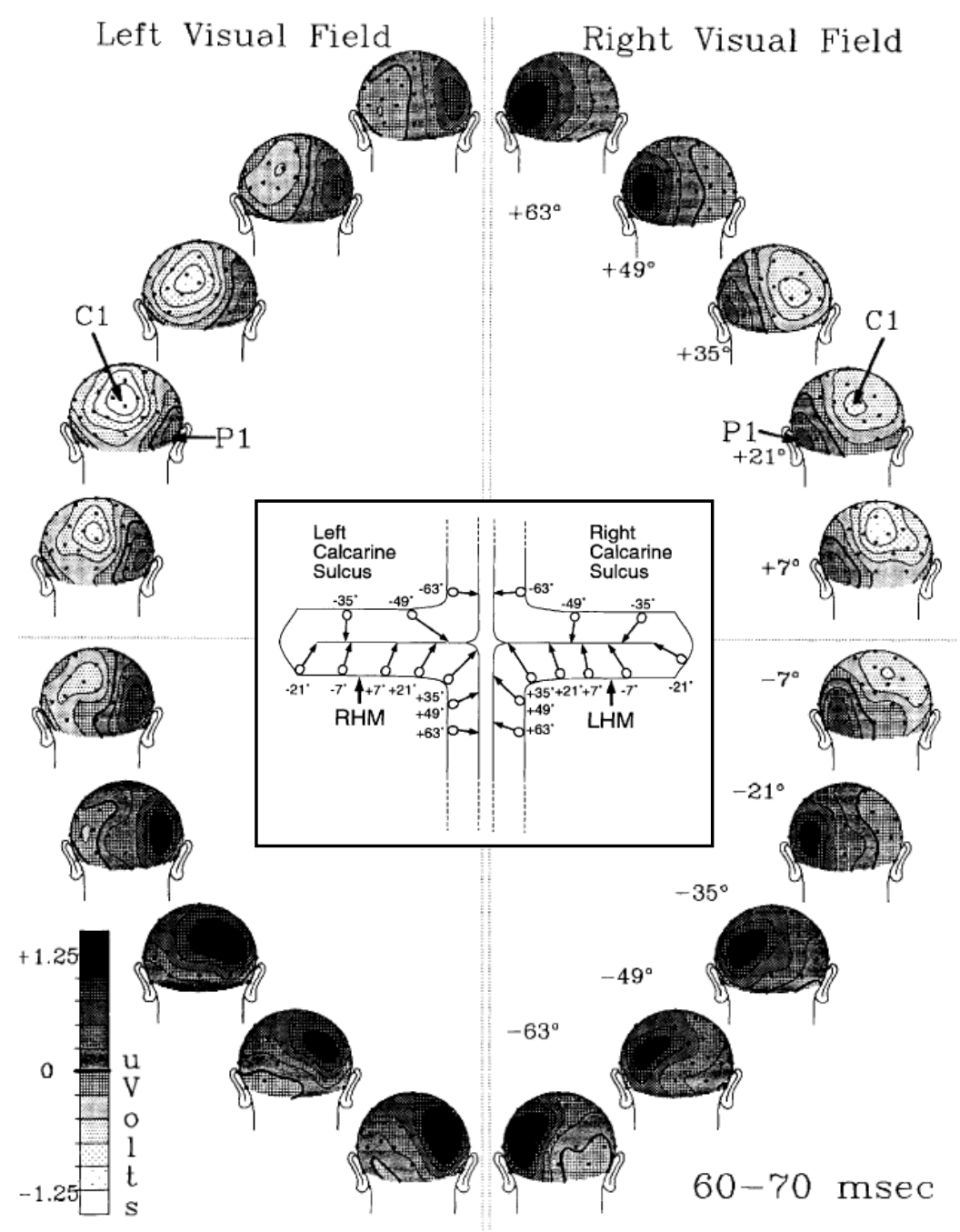



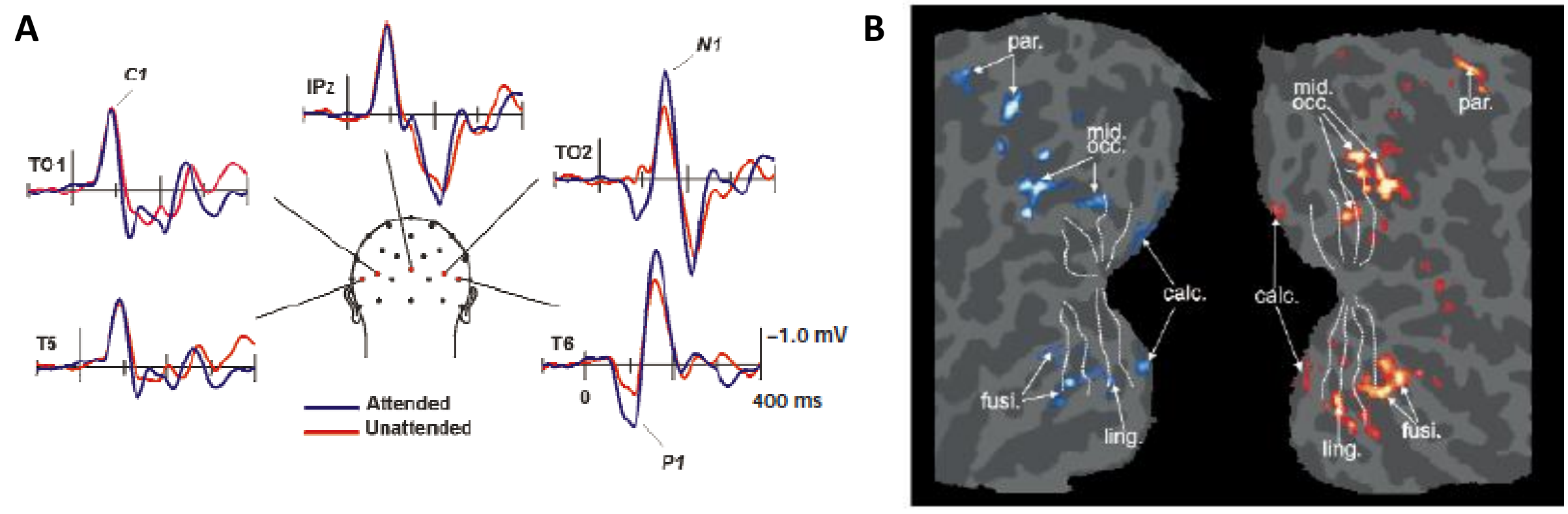


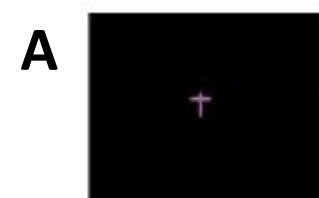

no stim.

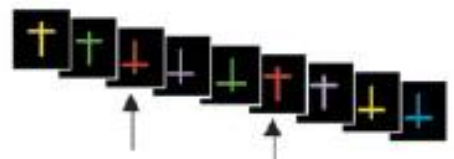

low load (easy task)

B
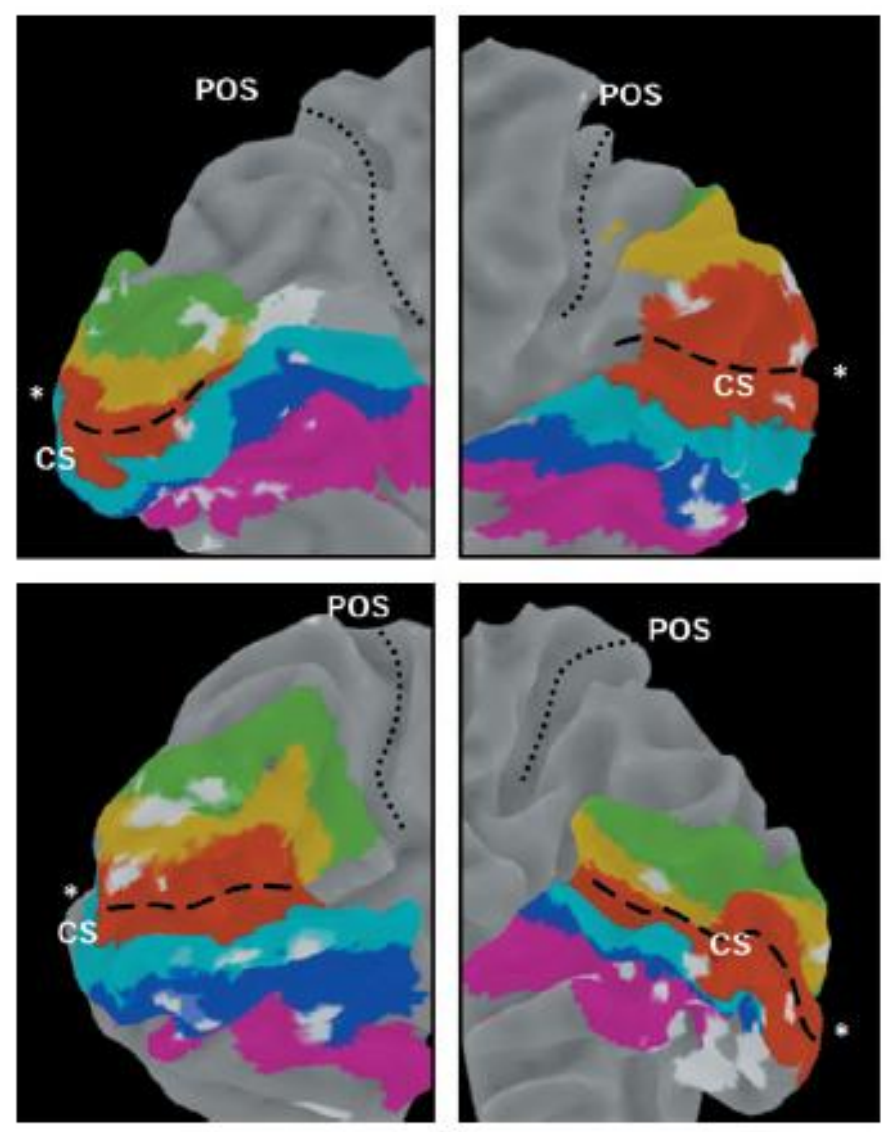

unilat. right

bilat.

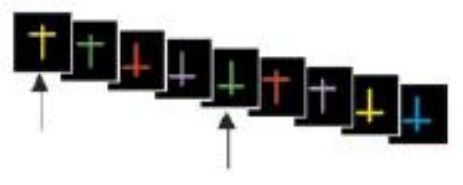

high load (difficuit task) 

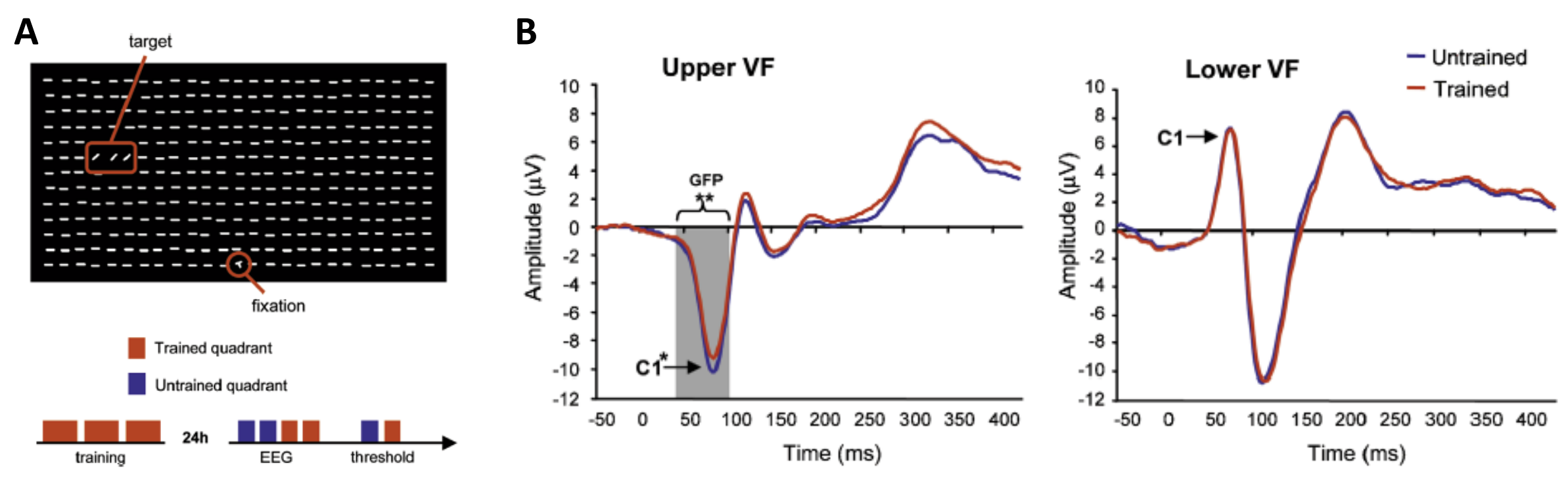
A
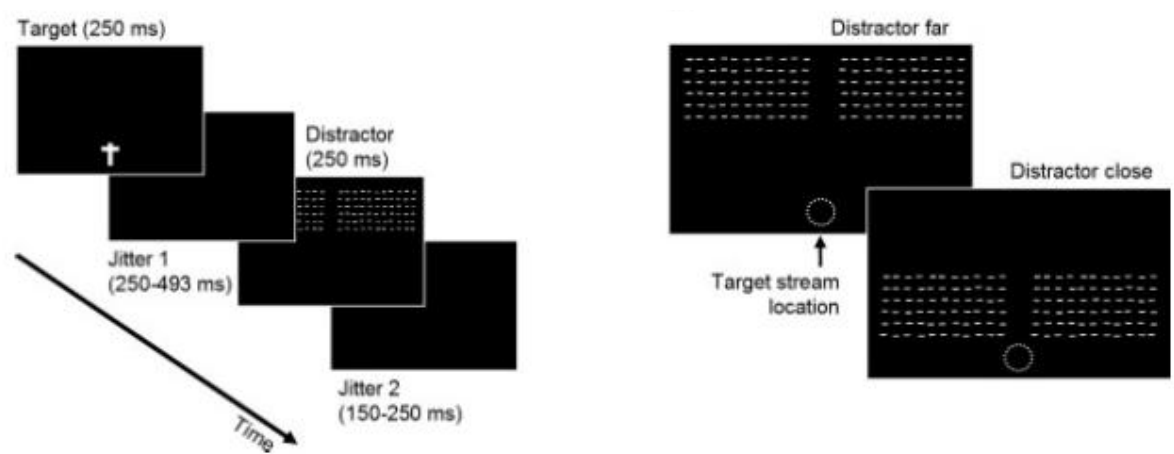

B

upper VF

lower VF

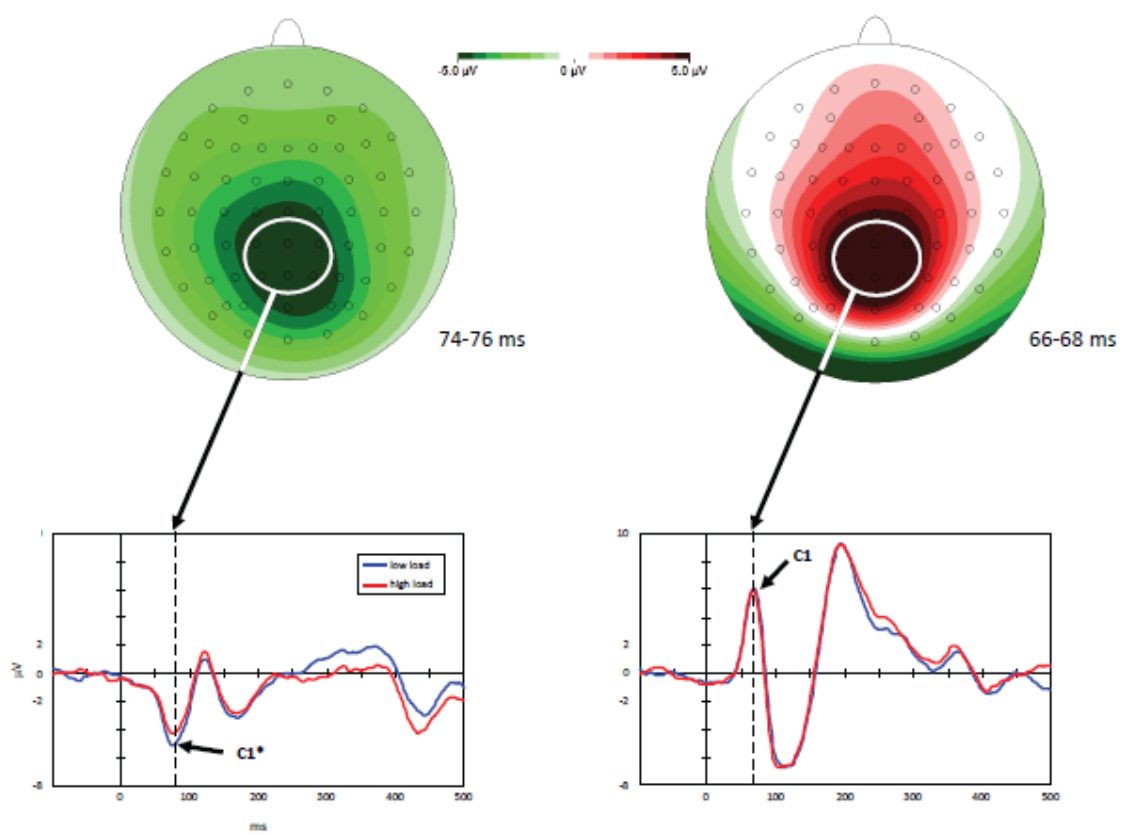


— stimulus 1

stimulus 2

stimulus 1 element

stimulus 2 element

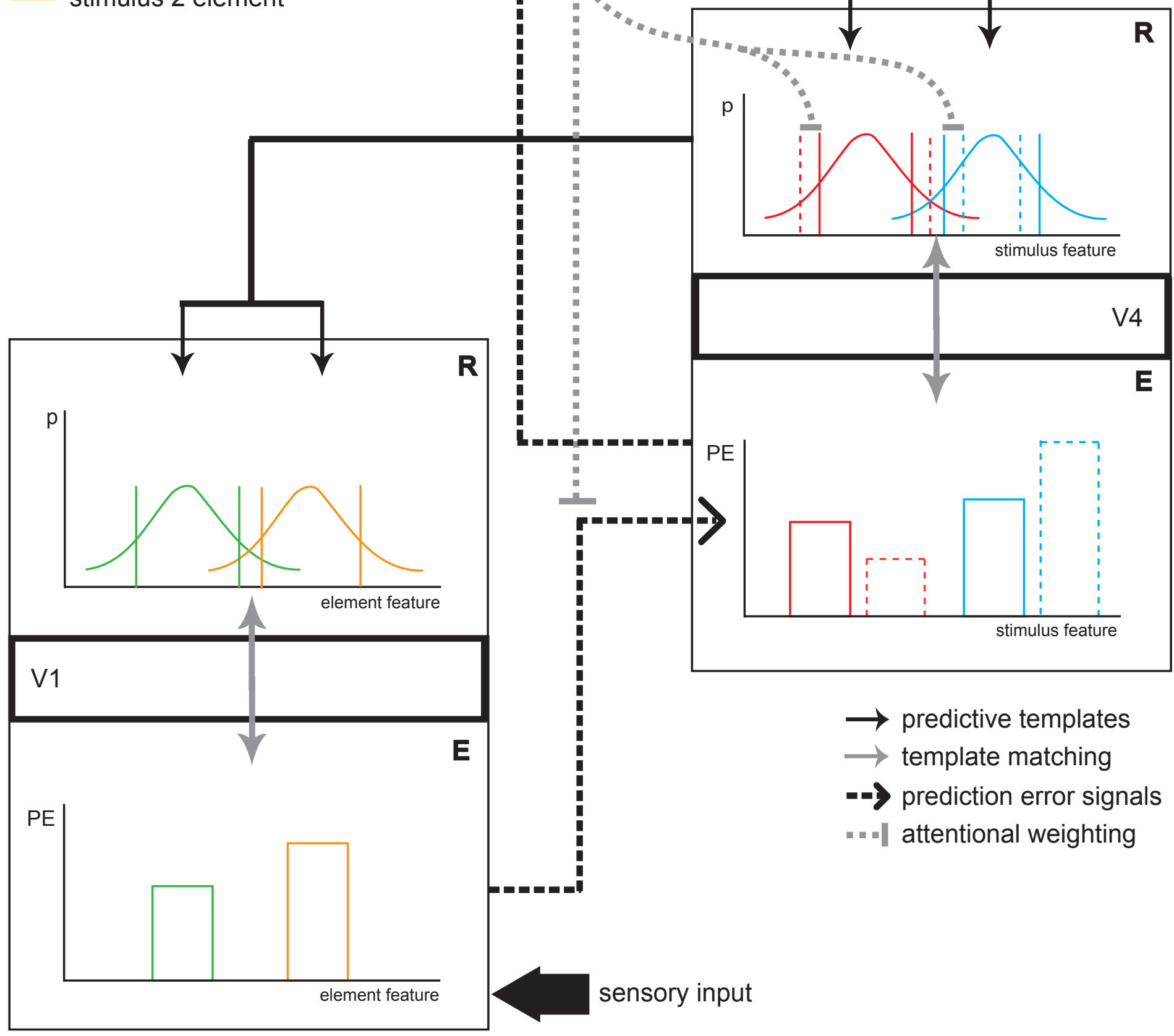

